\title{
Interaction effect on adiabatic pump of charge and spin in quantum dot
}

\author{
Satoshi Nakajima, Masahiko Taguchi, Toshihiro Kubo, and Yasuhiro Tokura* \\ Graduate School of Pure and Applied Sciences, University of Tsukuba, 1-1-1, Tennodai, Tsukuba 305-8571, Japan
}

(Received 25 January 2015; revised manuscript received 28 May 2015; published 19 November 2015)

\begin{abstract}
We investigate the pumped charge and spin at zero bias by a modulation of two control parameters using the full counting statistics with quantum master equation approach. First we study higher order effects of the pumping frequency in general Markov systems and show in this limit the equivalence between our approach and the real-time diagrammatic approach. An adiabatic modulation of the control parameters induces the BerrySinitsyn-Nemenman (BSN) phase. We show that the origin of the BSN phase is a nonadiabatic effect. The pumped charge (spin) is given by a summation of (i) a time integral of the instantaneous steady charge (spin) current and (ii) a geometric surface integral of the BSN curvature, which results from the BSN phase. In quantum dots (QDs) weakly coupled to two leads, we show that (i) is usually dominant if the thermodynamic parameters are modulated, although it is zero if the thermodynamic parameters are fixed to zero bias. To observe the spin effects, we consider collinear magnetic fields, which relate to spins through the Zeeman effect, with different amplitudes applying to the QDs and the leads. For interacting one level QD, we calculate analytically the pumped charge and spin by modulating the magnetic fields and the coupling strengths to the leads in the noninteracting and strong interacting limits. We show that the difference between these two limits appears through the instantaneous averages of the numbers of the electron with up and down spin in the QD. For the quantum pump by the modulation of the magnetic fields of the QD and one lead, the energy dependences of linewidth functions, which are usually neglected, are essential.
\end{abstract}

DOI: 10.1103/PhysRevB.92.195420

PACS number(s): 05.60.Gg, 72.25.-b, 73.63.Kv, 05.70.Ln

\section{INTRODUCTION}

In a mesoscopic system, even at zero bias, a charge or spin current is induced by a slow modulation of two or more control parameters [1-11]. This phenomenon, called quantum adiabatic pump, is theoretically interesting because its origins are quantum effects and nonequilibrium effects. The quantum adiabatic pump is also expected to be applied to the single electron transfer devices and the current standard [12,13].

The adiabatically pumped quantity is described by a geometric expression in the control parameter space, although the pumped quantity coming from second or more higher order of the pumping frequency is not geometric. In noninteracting systems, the quantum adiabatic pump had extensively been studied by the Brouwer formula [14-22], which describes the pumped charge by the scattering matrix. On the other hand, it is difficult to calculate the scattering matrix in the interacting systems. In the interacting system, the Brouwer formula had only been applied in mean field treatments [23,24] or in the Toulouse limit [25].

Recently, the quantum pump in interacting systems have been actively researched. There are three theoretical approaches. The first is the Green's function approach to pumping [26-28]. The second is the real-time diagrammatic approach [29-36] (RT approach), which uses the generalized master equation (GME) that is equivalent [37,38] to the quantum master equation (QME) derived using the Nakajima-Zwanzig projection operator technique [39]. Particularly, Ref. [34] derived a geometric expression similar to the Brouwer formula and the Berry-Sinitsyn-Nemenman (BSN) vector explained later. The third is the full counting statistics [40-42] (FCS) with the quantum master equation (FCS-QME, which is also

*tokura.yasuhiro.ft@u.tsukuba.ac.jp called generalized quantum master equation [42]) approach proposed in Ref. [43].

The adiabatic modulation of the control parameters induces a Berry-phase-like [44] quantity called BSN phase in the FCS-QME with the Markov approximation. Sinitsyn and Nemenman [45] studied the adiabatically pumped charge using the FCS and had shown that it is characterized by the BSN vector, which results from the BSN phase. The BSN vector was applied to the spin boson system [46] and a connection was made to the excess entropy production $[47,48]$. The FCS-QME approach can treat the Coulomb interaction, which cannot be treated in the Brouwer formula. The derived formula of the BSN vector depends on the approximations used for the QME. The Born-Markov approximation with or without the rotating wave approximation [39] (RWA) is frequently used. The QME in the Born-Markov approximation without RWA sometimes violates the non-negativity of the system reduced density operator [49]. The QME of the RWA or the coarse-graining approximation [50,51] (CGA) is the Lindblad type which guarantees the non-negativity [39].

Some recent papers $[33,34,43]$ showed that the Coulomb interaction induces the quantum pump. In Refs. [33,34], it was shown that in a one level interacting quantum dot (QD) weakly coupled to two leads, the pumped charge (also spin in Ref. [34]) induced by an adiabatic modulation of the energy level of the QD and the bias between the two leads vanishes in the noninteracting limit. In particular, Yuge et al. [43] studied the pumped charge coming from the BSN curvatures by adiabatic modulation of the thermodynamic parameters (the chemical potentials and the temperatures) in spinless QDs weakly coupled to two spinless leads and showed that the BSN curvatures are zero in noninteracting QDs although they are nonzero for finite interaction.

In this paper, we first generalize the FCS-QME approach to multicounting field to calculate spin current (Sec. II A). We 
then study the nonadiabatic effects in general Markov systems and clarified the relations between the FCS-QME approach and the RT approach [34] in Sec. II B. Additionally we show that the origin of the BSN phase is a nonadiabatic effect. Next we explain the model to be considered (Sec. III). We consider QDs weakly coupled to two leads ( $L$ and $R$ ). To observe the spin effects, we consider collinear magnetic fields, which relate to spins through the Zeeman effect, with different amplitudes applying to the QDs $\left(B_{S}\right)$ and the leads $\left(B_{L}\right.$ and $\left.B_{R}\right)$. The dynamic parameters $\left(B_{S}, B_{L / R}\right.$, and the coupling strengths to the leads) are control parameters. We use the RWA defined as a long coarse-graining time limit of the CGA to the FCS-QME. In Sec. IV and Sec. V, we consider noninteracting and interacting QDs, respectively. First, we show (in Sec. IV B and Sec. VB) the time integral of an instantaneous steady current is usually dominant if the thermodynamic parameters (the chemical potentials and the temperatures of leads) are modulated (as considered in Refs. [33,34,43,52,53]). Next in a one level QD with the Coulomb interaction $U$, we analytically calculate the BSN curvatures of spin and charge induced by the dynamic parameters in the noninteracting (Sec. IV C) and strong (Sec. V C) interacting limit $(U \rightarrow 0, \infty)$. The difference between the results for $U=0$ and $U=\infty$ appears through the instantaneous averages of the numbers of the electron with up and down spin in the QD. For the quantum pump by the adiabatic modulation of $\left(B_{L}, B_{S}\right)$, the energy dependences of linewidth functions, which are usually neglected, are essential. In Sec. V C, we show and discuss the contour plots of BSN curvatures evaluated numerically. Finally, we summarize this paper with discussions (Sec. VI). In Appendix A, the Liouville space $[42,54]$ and the matrix representation of the Liouvillian are explained. In Appendix B, we derive the FCS-QME of the CGA and discuss the difference between the CGA and the RWA. In Appendix C, we derive Eq. (5).In Appendix D, we discuss the validity of the adiabatic expansion in Sec. II B. In Appendix E, we discuss the derivation of Eq. (31).

\section{FCS-QME}

In this section, we consider general Markov systems weakly coupled to noninteracting (fermionic or bosonic) baths. The model we use to do a concrete calculation is explained at Sec. III.

In Sec. II A, we explain the FCS-QME method using the Liouville space [42,54] (Appendix A). This method is a generalization to the multicounting field of Ref. [43]. In Sec. IIB, we study nonadiabatic effect, and show the equivalence to the method of Ref. [34].

\section{A. Derivation of FCS-QME}

Consider a cyclic modulation of the control parameters with a period $\tau$. At $t=0$ and $t=\tau$, we perform projection measurements of $\mu$ th time-independent observables $\left\{O_{\mu}\right\}$ indexed by $\mu$ of baths which commute with each other. $\Delta o_{\mu}=o_{\mu}^{(\tau)}-o_{\mu}^{(0)}$ denotes the difference of the outcomes $\left\{o_{\mu}^{(\tau)}\right\}$ at $t=\tau$ and the outcomes $\left\{o_{\mu}^{(0)}\right\}$ at $t=0$. The Fourier transform of the joint probability density distribution $P_{\tau}\left(\left\{\Delta o_{\mu}\right\}\right), Z_{\tau}\left(\left\{\chi_{\mu}\right\}\right)=$ $\int \prod_{\mu} d \Delta o_{\mu} P_{\tau}\left(\left\{\Delta o_{\mu}\right\}\right) e^{i \sum_{\mu} \chi_{\mu} \Delta o_{\mu}}$, is the generating function. Here, $\chi_{\mu}$ are counting fields for $O_{\mu} . Z_{\tau}\left(\left\{\chi_{\mu}\right\}\right)$ is given by $Z_{\tau}\left(\left\{\chi_{\mu}\right\}\right)=\operatorname{Tr}_{\text {tot }}\left[\rho_{\text {tot }}^{\chi}(t=\tau)\right]$ using an operator of the total system $\rho_{\text {tot }}^{\chi}(t)$ [42]. Here, $\chi$ denotes the set of the counting fields $\left\{\chi_{\mu}\right\}$. We defined $\rho^{\chi}(t) \stackrel{\text { def }}{=} \operatorname{Tr}_{B}\left[\rho_{\text {tot }}^{\chi}(t)\right]$, where $\operatorname{Tr}_{B}$ denotes a trace over baths' degrees of freedom. $\rho^{\chi}(t)$ provides the generating function $Z_{\tau}\left(\left\{\chi_{\mu}\right\}\right)=\operatorname{Tr}_{S}\left[\rho^{\chi}(t=\tau)\right]$. In Appendix B, we derive the full counting statistics with quantum master equation (FCS-QME) [i.e., the equation of motion of $\left.\rho^{\chi}(t)\right]$ from the equation of motion of $\rho_{\mathrm{tot}}^{\chi}(t)$. In this paper, we set $\hbar=1$. We suppose $\rho_{\text {tot }}(0)=\rho(0) \otimes \rho_{B}\left(\alpha_{0}\right)$ and $\rho_{\text {tot }}(t) \approx \rho(t) \otimes \rho_{B}\left(\alpha_{t}\right)(0<t \leqslant \tau)$, where $\rho_{\text {tot }}(t)$ is the total system state, $\rho(t)=\operatorname{Tr}_{B}\left[\rho_{\text {tot }}(t)\right]$ is the system reduced density operator, $\rho_{B}\left(\alpha_{t}\right)$ is a tensor product of the grand canonical or canonical distributions of the baths, and $\alpha_{t}$ is the value of the set of the control parameters at time $t$. If the baths are electric leads, $\rho_{B}\left(\alpha_{t}\right)$ is given by Eq. (B2). The FCS-QME [42,43] is

$$
\frac{d \rho^{\chi}(t)}{d t}=\hat{K}^{\chi}\left(\alpha_{t}\right) \rho^{\chi}(t),
$$

and the initial condition is $\rho^{\chi}(0)=\rho(0)$. Here $\hat{K}^{\chi}\left(\alpha_{t}\right)$ is the Liouvillian modified by $\chi$. The Liouvillian depends on used approximations, for instance, the Born-Markov approximation without or within RWA [39] and the CGA [50,51]. After Sec. III we choose the Born-Markov approximation within RWA; however, in this section we assume only Markov property (i.e., $\hat{K}^{\chi}$ just depends on $\alpha_{t}$ ). At $\chi=0$, the FCS$\mathrm{QME}$ becomes the quantum master equation (QME)

$$
\frac{d \rho(t)}{d t}=\hat{K}\left(\alpha_{t}\right) \rho(t) .
$$

$\hat{K}\left(\alpha_{t}\right)$ equals $\hat{K}^{\chi}\left(\alpha_{t}\right)$ at $\chi=0$. In the following, a symbol $X$ without $\chi$ denotes $\left.X^{\chi}\right|_{\chi=0}$.

In the Liouville space (Appendix A), the left and right eigenvalue equations of the Liouvillian are

$$
\begin{aligned}
\hat{K}^{\chi}(\alpha)\left|\rho_{n}^{\chi}(\alpha)\right\rangle & =\lambda_{n}^{\chi}(\alpha)\left|\rho_{n}^{\chi}(\alpha)\right\rangle, \\
\left\langle\left\langle l_{n}^{\chi}(\alpha)\right| \hat{K}^{\chi}(\alpha)\right. & =\lambda_{n}^{\chi}(\alpha) \mid\left\langle l_{n}^{\chi}(\alpha)\right| .
\end{aligned}
$$

The left eigenvectors $l_{n}^{\chi}(\alpha)$ and the right eigenvectors $\rho_{m}^{\chi}(\alpha)$ (operators considered as elements of a vector space) satisfy $\left\langle\left\langle l_{n}^{\chi}(\alpha) \mid \rho_{m}^{\chi}(\alpha)\right\rangle\right\rangle=\delta_{n m}$. Here, $\alpha$ denotes arbitrary values of the set of the control parameters. The mode which has the eigenvalue with the maximum real part is assigned by the label $n=0$ and is called the slowest mode. In the limit $\chi \rightarrow 0, \lambda_{0}^{\chi}(\alpha)$ becomes 0 and $\left\langle\left\langle l_{0}^{\chi}(\alpha)\right|\right.$ becomes $\left\langle\langle 1|\right.$, i.e., $l_{0}(\alpha)=1$. The conservation of the probability $\frac{d}{d t}\langle\langle 1 \mid \rho(t)\rangle\rangle=\left\langle\left\langle 1\left|\hat{K}\left(\alpha_{t}\right)\right| \rho(t)\right\rangle\right\rangle=0$ leads $\langle\langle 1| \hat{K}(\alpha)=0$. In addition, $n=0$ mode right eigenvector, $\left.\left|\rho_{0}(\alpha)\right\rangle\right\rangle$, determined by $\left.\hat{K}(\alpha)\left|\rho_{0}(\alpha)\right\rangle\right\rangle=0$, represents the instantaneous steady state; if the control parameters are fixed to $\alpha$, the state $\rho(t)$ converges to $\rho_{0}(\alpha)$ at $t \rightarrow \infty$. In general, the solution of the FCS-QME Eq. (1) is expanded as

$$
\left.\left.\left|\rho^{\chi}(t)\right\rangle\right\rangle=\sum_{n} c_{n}^{\chi}(t) e^{\Lambda_{n}^{\chi}(t)}\left|\rho_{n}^{\chi}\left(\alpha_{t}\right)\right\rangle\right\rangle,
$$

where $\Lambda_{n}^{\chi}(t)=\int_{0}^{t} d s \lambda_{n}^{\chi}\left(\alpha_{s}\right)$. The coefficients $c_{n}^{\chi}(t)$ obey Eq. (C8). The coefficients $c_{n}^{\chi}(t)$ are given by solving Eq. (C8). The condition which makes $\left\{c_{n}^{\chi}(t)\right\}_{n \neq 0}$ negligible is discussed in Appendix C and was studied carefully in Ref. [55] for $\chi=0$. 
In this section, we consider sufficiently slow modulation of the control parameters. The effects of fast modulation are considered in the next section. For the slowest mode, the second term of the right side of Eq. (C8) exponentially damps as a function of time. The relaxation time of the system $\left(\tau_{S}\right)$ is the order of $\Gamma^{-1}$, where $\Gamma$ is the typical value of the linewidth functions [defined as $\Gamma_{i}$ in Eq. (58)]. Assuming the cycle time $\tau$ is much longer than $\tau_{S}$, we obtain

$$
c_{0}^{\chi}(\tau)=c_{0}^{\chi}(0) \exp \left[-\int_{0}^{\tau} d t\left\langle\left\langle l_{0}^{\chi}\left(\alpha_{t}\right)\left|\frac{d}{d t}\right| \rho_{0}^{\chi}\left(\alpha_{t}\right)\right\rangle\right\rangle\right]
$$

and

$$
c_{n}^{\chi}(\tau) e^{\Lambda_{n}^{\chi}(\tau)} \approx 0(n \neq 0) .
$$

In fact, $c_{n}^{\chi}(t) e^{\Lambda_{n}^{\chi}(t)}=O\left(\frac{\omega}{\Gamma}\right)$ with $\omega=2 \pi / \tau$ as explained in Appendix $C$. We denote the condition which makes the above approximation appropriate as $\chi$-adiabatic condition. Using the initial condition $\rho^{\chi}(0)=\rho(0)$, we obtain $c_{0}^{\chi}(0)=$ $\left\langle\left\langle l_{0}^{\chi}\left(\alpha_{0}\right) \mid \rho(0)\right\rangle\right\rangle$. Substituting these equations into Eq. (5), we obtain [43]

$$
\begin{aligned}
\left.\left|\rho^{\chi}(\tau)\right\rangle\right\rangle \approx & \left\langle\left\langle l_{0}^{\chi}\left(\alpha_{0}\right) \mid \rho(0)\right\rangle\right\rangle e^{-\int_{0}^{\tau} d t\left\langle\left\langle l_{0}^{\chi}\left(\alpha_{t}\right)\left|\frac{d}{d t}\right| \rho_{0}^{\chi}\left(\alpha_{t}\right)\right\rangle\right\rangle} \\
& \left.\times e^{\int_{0}^{\tau} d t \lambda_{0}^{\chi}\left(\alpha_{t}\right)}\left|\rho_{0}^{\chi}\left(\alpha_{\tau}\right)\right\rangle\right\rangle,
\end{aligned}
$$

and the cumulant generating function $S_{\tau}(\chi)=\ln Z_{\tau}(\chi)=$ $\ln \left\langle\left\langle 1 \mid \rho^{\chi}(\tau)\right\rangle\right\rangle$ :

$$
\begin{aligned}
S_{\tau}(\chi)= & \int_{0}^{\tau} d t \lambda_{0}^{\chi}\left(\alpha_{t}\right)-\oint_{C} d \alpha^{n}\left\langle\left\langle l_{0}^{\chi}(\alpha)\right| \frac{\left.\partial\left|\rho_{0}^{\chi}(\alpha)\right\rangle\right\rangle}{\partial \alpha^{n}}\right. \\
& +\ln \left\langle\left\langle l_{0}^{\chi}\left(\alpha_{0}\right) \mid \rho(0)\right\rangle\right\rangle+\ln \left\langle\left\langle 1 \mid \rho_{0}^{\chi}\left(\alpha_{\tau}\right)\right\rangle\right\rangle .
\end{aligned}
$$

Here, we used $\int_{0}^{\tau} d t\left\langle\left\langle l_{0}^{\chi}\left(\alpha_{t}\right)\left|\frac{d}{d t}\right| \rho_{0}^{\chi}\left(\alpha_{t}\right)\right\rangle\right\rangle=\oint_{C} d \alpha^{n}\left\langle\left\langle l_{0}^{\chi}(\alpha)\right|\right.$ $\frac{\left.\partial\left|\rho_{0}^{\chi}(\alpha)\right\rangle\right\rangle}{\partial \alpha^{n}}$, where $C$ is the trajectory from $\alpha_{0}$ to $\alpha_{\tau}, \alpha^{n}$ are the $n$th component of the control parameters, and the summation symbol $\sum_{n}$ is omitted. Equation (9) is the same with Yuge et al. [43] except that $\chi$ denotes a multicounting field. The averages $\left\langle\Delta o_{\mu}\right\rangle_{\tau}=\left.\frac{\partial S_{\tau}(\chi)}{\partial\left(i \chi_{\mu}\right)}\right|_{\chi=0}$ are

$$
\begin{aligned}
\left\langle\Delta o_{\mu}\right\rangle_{\tau}= & \int_{0}^{\tau} d t \lambda_{0}^{\mu}\left(\alpha_{t}\right)-\oint_{C} d \alpha^{n}\left\langle\left\langle l_{0}^{\mu}(\alpha)\right| \frac{\left.\partial\left|\rho_{0}(\alpha)\right\rangle\right\rangle}{\partial \alpha^{n}}\right. \\
& +\left\langle\left\langle l_{0}^{\mu}\left(\alpha_{0}\right) \mid \rho(0)\right\rangle\right\rangle+\left\langle\left\langle 1 \mid \rho_{0}^{\mu}\left(\alpha_{0}\right)\right\rangle\right\rangle
\end{aligned}
$$

where $\left.X^{\mu}(\alpha) \stackrel{\text { def }}{=} \frac{\partial X^{\chi}(\alpha)}{\partial\left(i \chi_{\mu}\right)}\right|_{\chi=0}$. Here, we used $-\oint_{C} d \alpha^{n}\left\langle\left\langle l_{0}(\alpha)\right.\right.$ $\mid \frac{\left.\partial\left|\rho_{0}^{\mu}(\alpha)\right\rangle\right\rangle}{\partial \alpha^{n}}=-\left\langle\left\langle 1 \mid \rho_{0}^{\mu}\left(\alpha_{\tau}\right)\right\rangle\right\rangle+\left\langle\left\langle 1 \mid \rho_{0}^{\mu}\left(\alpha_{0}\right)\right\rangle\right\rangle$ because $\left\langle\left\langle l_{0}(\alpha)\right|\right.$ $\frac{\left.\partial\left|\rho_{0}^{\mu}(\alpha)\right\rangle\right\rangle}{\partial \alpha^{n}}=\frac{\partial}{\partial \alpha^{n}}\left\langle\left\langle 1 \mid \rho_{0}^{\mu}(\alpha)\right\rangle\right\rangle$. The integrand of the first time integral, $\lambda_{0}^{\mu}\left(\alpha_{t}\right)$, are the instantaneous steady currents of $O_{\mu}$ at time $t$; if the control parameters are fixed to $\alpha$ and the state is $\rho_{0}(\alpha)$, the current of $O_{\mu}$ is $\lambda_{0}^{\mu}(\alpha)$. The integrand of the second term of the right side of Eq. (10) is the Berry-Sinitsyn-Nemenman (BSN) vector [45]

$$
A_{n}^{\mu}(\alpha)=\left\langle\left\langle l_{0}^{\mu}(\alpha)\right| \frac{\left.\partial\left|\rho_{0}(\alpha)\right\rangle\right\rangle}{\partial \alpha^{n}} .\right.
$$

The third and fourth terms of the right side of Eq. (10) cancel if the initial condition is the instantaneous steady state $\rho_{0}\left(\alpha_{0}\right)$. Because of $\alpha_{\tau}=\alpha_{0}$, the second term of the right side of Eq. (10) can be described as a surface integral over the surface
$S$ enclosed by $C$ using the Stokes theorem:

$$
\begin{gathered}
\left\langle\Delta o_{\mu}\right\rangle_{\tau}=\left\langle\Delta o_{\mu}\right\rangle_{\tau}^{\text {Steady }}+\left\langle\Delta o_{\mu}\right\rangle_{S}^{\text {Berry }}, \\
\left\langle\Delta o_{\mu}\right\rangle_{\tau}^{\text {Steady }}=\int_{0}^{\tau} d t \lambda_{0}^{\mu}\left(\alpha_{t}\right), \\
\left\langle\Delta o_{\mu}\right\rangle_{S}^{\text {Berry }}=-\int_{S} d \alpha^{m} \wedge d \alpha^{n} \frac{1}{2} F_{m n}^{\mu}(\alpha) .
\end{gathered}
$$

Here, $\wedge$ is the wedge product and the summation symbol $\sum_{n, m}$ is omitted. BSN curvature $F_{m n}^{\mu}(\alpha)$ is given by

$$
F_{m n}^{\mu}(\alpha)=\frac{\partial A_{n}^{\mu}(\alpha)}{\partial \alpha^{m}}-\frac{\partial A_{m}^{\mu}(\alpha)}{\partial \alpha^{n}} .
$$

Yuge et al. [43] focus on only the second term of Eq. (12) subtracting the first term, and they did not evaluate $\left\langle\Delta o_{\mu}\right\rangle_{\tau}^{\text {Steady }}$. In Sec. IV B, we show that this contribution is usually dominant if the thermodynamic parameters are modulated although the steady currents $\lambda_{0}^{\mu}\left(\alpha_{t}\right)$ are zero if the thermodynamic parameters are fixed to zero bias.

From the cumulant generating function Eq. (9), we can calculate the second order cumulants ${ }^{c}\left\langle o_{\mu} o_{\nu}\right\rangle_{\tau}=\left.\frac{\partial^{2} S_{\tau}(\chi)}{\partial\left(i \chi_{\mu}\right) \partial\left(i \chi_{\nu}\right)}\right|_{\chi=0}$ and higher cumulants. However, we focus only on the first order cumulants (averages) in this paper. Up to the first order cumulants, we do not need the multicounting field. In fact, the multicounting field is helpful to understand the physical origin of each term of the (FCS-)QME.The counting fields appear only in $A \bullet B(A, B \neq 1)$ type terms in the dissipator term $\hat{\Pi}^{\chi} \bullet$ [see Eq. (A7); • is an arbitrary operator] as Eq. (B7). For instance, the factor $e^{i \chi_{b \sigma}}$ with the counting field for total number of electron with spin $\sigma$ of bath $b, \chi_{b \sigma}$, means tunneling process from the system to bath $b$ with $\operatorname{spin} \sigma$. The factor $e^{-i \chi_{b \sigma}}$ means tunneling process from bath $b$ with spin $\sigma$ to the system.

\section{B. Nonadiabatic effect and BSN vector}

In this section, we consider nonadiabatic effect [that comes from $\left(\frac{\omega}{\Gamma}\right)^{n}(n=1,2, \ldots)$ with $\left.\omega=2 \pi / \tau\right]$, which had been researched recently $[31,35,52,53]$. If the modulation of the control parameters is not adiabatic, the difference between the state and the instantaneous steady state, $\rho^{a}(t) \stackrel{\text { def }}{=} \rho(t)-$ $\rho_{0}\left(\alpha_{t}\right)=\sum_{n \neq 0} c_{n}(t) e^{\Lambda_{n}(t)} \rho_{n}\left(\alpha_{t}\right)$, is important. In contrast to Appendix $C$ and Ref. [55], we do not treat $\left\{c_{n}(t)\right\}_{n \neq 0}$ explicitly; instead, we use the pseudoinverse of the Liouvillian. The formal solution of the FCS-QME Eq. (1) is

$$
\left.\left.\left|\rho^{\chi}(t)\right\rangle\right\rangle=\mathrm{T} \exp \left[\int_{0}^{t} d s \hat{K}^{\chi}\left(\alpha_{s}\right)\right]\left|\rho^{\chi}(0)\right\rangle\right\rangle,
$$

where $\mathrm{T}$ denotes the time-ordering operation. Using this, we obtain the averages [52]

$$
\begin{aligned}
\left\langle\Delta o_{\mu}\right\rangle_{t} & =\left.\frac{\partial}{\partial\left(i \chi_{\mu}\right)}\left\langle\left\langle 1 \mid \rho^{\chi}(t)\right\rangle\right\rangle\right|_{\chi=0} \\
& =\int_{0}^{t} d u\left\langle\left\langle 1\left|\hat{K}^{\mu}\left(\alpha_{u}\right)\right| \rho(u)\right\rangle\right\rangle+\left\langle\left\langle 1 \mid \rho^{\mu}(0)\right\rangle\right\rangle \\
& =\int_{0}^{t} d u\left\langle\left\langle 1\left|\hat{K}^{\mu}\left(\alpha_{u}\right)\right| \rho(u)\right\rangle\right\rangle \equiv \int_{0}^{t} d u I_{\mu}(u) .
\end{aligned}
$$


Here, we use $\left\langle\langle 1| \hat{K}(\alpha)=0\right.$ in the second line and $\left.\left.\mid \rho^{\mu}(0)\right\rangle\right\rangle=0$ (derived from $\left.\left.\left|\rho^{\chi}(0)\right\rangle\right\rangle=|\rho(0)\rangle\right\rangle$ ) in the third line. Moreover, using $\left\langle\left\langle l_{0}(\alpha)\right|=\left\langle\langle 1|, \lambda_{0}(\alpha)=0\right.\right.$, and Eq. (4), we obtain [53]

$$
\left\langle\langle 1| \hat{K}^{\mu}(\alpha)=\lambda_{0}^{\mu}(\alpha)\left\langle\langle 1|-\left\langle\left\langle l_{0}^{\mu}(\alpha)\right| \hat{K}(\alpha) .\right.\right.\right.
$$

Hence the currents $I_{\mu}(t)$ are given by

$$
\begin{aligned}
I_{\mu}(t) & =\left\langle\left\langle 1\left|\hat{K}^{\mu}\left(\alpha_{t}\right)\right| \rho(t)\right\rangle\right\rangle \\
& =\lambda_{0}^{\mu}\left(\alpha_{t}\right)-\left\langle\left\langle l_{0}^{\mu}\left(\alpha_{t}\right)\left|\hat{K}\left(\alpha_{t}\right)\right| \rho(t)\right\rangle\right\rangle \\
& =\lambda_{0}^{\mu}\left(\alpha_{t}\right)-\left\langle\left\langle l_{0}^{\mu}\left(\alpha_{t}\right)\left|\frac{d}{d t}\right| \rho(t)\right\rangle\right\rangle .
\end{aligned}
$$

The used approximation is only the Markov property of the Liouvillian, Eq. (1). In Appendix D, we discuss the reasonable range of the power of $\frac{\omega}{\Gamma}$ (nonadiabaticity). Substituting Eq. (19) with $\rho(t) \approx \rho_{0}\left(\alpha_{t}\right)$ into Eq. (17), we obtain Eq. (10) without the third and fourth terms. If $\rho(0) \neq \rho_{0}\left(\alpha_{0}\right)$, the state relaxes to the instantaneous steady state in the relaxation time $\tau_{S}$. The third and fourth terms of Eq. (10), $\left\langle\Delta o_{\mu}\right\rangle_{\tau}^{3+4}=\left\langle\left\langle l_{0}^{\mu}\left(\alpha_{0}\right) \mid \rho(0)\right\rangle\right\rangle+$ $\left\langle\left\langle 1 \mid \rho_{0}^{\mu}\left(\alpha_{0}\right)\right\rangle\right\rangle$, result from this relaxation. The contribution of $\left\langle\Delta o_{\mu}\right\rangle_{\tau}$ from $\delta \rho(0)=\rho^{a}(0)=\rho(0)-\rho_{0}\left(\alpha_{0}\right)$ is

$$
\begin{aligned}
\left\langle\Delta o_{\mu}\right\rangle_{\tau}^{\text {ini }} \stackrel{\text { def }}{=} & -\int_{0}^{\tau} d t\left\langle\left\langle l_{0}^{\mu}\left(\alpha_{t}\right)\left|\frac{d}{d t}\right| \delta \rho(t)\right\rangle\right\rangle \\
= & \left\langle\left\langle l_{0}^{\mu}\left(\alpha_{0}\right) \mid \delta \rho(0)\right\rangle\right\rangle-\left\langle\left\langle l_{0}^{\mu}\left(\alpha_{\tau}\right) \mid \delta \rho(\tau)\right\rangle\right\rangle \\
& \left.+\int_{0}^{\tau} d t \frac{d\left\langle\left\langle l_{0}^{\mu}\left(\alpha_{t}\right)\right|\right.}{d t}|\delta \rho(t)\rangle\right\rangle,
\end{aligned}
$$

with $\left.|\delta \rho(t)\rangle\rangle \stackrel{\text { def }}{=} \mathrm{T} \exp \left[\int_{0}^{t} d s \hat{K}\left(\alpha_{s}\right)\right]|\delta \rho(0)\rangle\right\rangle$. The first term of the right side of Eq. (20) is $\left\langle\Delta o_{\mu}\right\rangle_{\tau}^{3+4}$. Because we can obtain $\left\langle\left\langle l_{0}^{\mu}(\alpha) \mid \rho_{0}(\alpha)\right\rangle\right\rangle+\left\langle\left\langle 1 \mid \rho_{0}^{\mu}(\alpha)\right\rangle\right\rangle=0$ from the normalization $\left\langle\left\langle l_{0}^{\chi}(\alpha) \mid \rho_{0}^{\chi}(\alpha)\right\rangle\right\rangle=1,\left\langle\Delta o_{\mu}\right\rangle_{\tau}^{3+4}$ is given by $\left\langle\left\langle l_{0}^{\mu}\left(\alpha_{0}\right)[|\rho(0)\rangle\rangle-\right.\right.$ $\left.\left.\left|\rho\left(\alpha_{0}\right)\right\rangle\right\rangle\right]=\left\langle\left\langle l_{0}^{\mu}\left(\alpha_{0}\right) \mid \delta \rho(0)\right\rangle\right\rangle$. The second term of the right side of Eq. (20) is exponentially small since $\delta \rho(\tau) \sim e^{-\Gamma \tau}$. The order of the third term is $O\left(\frac{\omega}{\Gamma}\right)$ with $\omega=2 \pi / \tau$ because $\frac{d\left\langle\left\langle l_{0}^{\mu}\left(\alpha_{t}\right)\right|\right.}{d t}=O(\omega)$ and the integral range is restricted up to $1 / \Gamma$ since $\delta \rho(t) \sim e^{-\Gamma t}$. Hence $\left\langle\Delta o_{\mu}\right\rangle_{\tau}^{\text {ini }}=\left\langle\Delta o_{\mu}\right\rangle_{\tau}^{3+4}+O\left(\frac{\omega}{\Gamma}\right)$. The currents can also be written as

$$
I_{\mu}(t)=\left\langle\left\langle 1\left|W_{\mu}\left(\alpha_{t}\right)\right| \rho(t)\right\rangle\right\rangle,
$$

where $W_{\mu}(\alpha)$ are the current operators defined by

$$
\left\langle\langle 1| W_{\mu}(\alpha)=\left\langle\langle 1| \hat{K}^{\mu}(\alpha),\right.\right.
$$

i.e., $\operatorname{Tr}_{S}\left[W_{\mu}(\alpha) \bullet\right]=\operatorname{Tr}_{S}\left[\hat{K}^{\mu}(\alpha) \bullet\right]$ for any operator $\bullet$ [see Eq. (A14)]. Using Eq. (18), the instantaneous steady currents are given by

$$
\left\langle\left\langle 1\left|W_{\mu}(\alpha)\right| \rho_{0}(\alpha)\right\rangle\right\rangle=\lambda_{0}^{\mu}(\alpha)=I_{\mu}^{\text {Steady }}(\alpha) .
$$

In the QDs weakly coupled to two leads, the electric current operator [i.e., $W_{\mu}(\alpha)$ corresponding to the electric current] coincides with Ref. [56] in the Born-Markov approximation without or within RWA.

Applying the pseudoinverse $\mathcal{R}(\alpha)$ defined by

$$
\left.\mathcal{R}(\alpha) \hat{K}(\alpha)=1-\left|\rho_{0}(\alpha)\right\rangle\right\rangle\langle\langle 1|
$$

to the QME Eq. (2), we obtain

$$
\begin{aligned}
\left.\left|\rho^{a}(t)\right\rangle\right\rangle & \left.\left.=\mathcal{R}\left(\alpha_{t}\right) \frac{d}{d t}\left|\rho_{0}\left(\alpha_{t}\right)\right\rangle\right\rangle+\mathcal{R}\left(\alpha_{t}\right) \frac{d}{d t}\left|\rho^{a}(t)\right\rangle\right\rangle \\
& \left.\left.=\sum_{n=1}^{\infty}\left[\mathcal{R}\left(\alpha_{t}\right) \frac{d}{d t}\right]^{n}\left|\rho_{0}\left(\alpha_{t}\right)\right\rangle\right\rangle \equiv \sum_{n=1}^{\infty}\left|\rho^{a(n)}(t)\right\rangle\right\rangle .
\end{aligned}
$$

Substituting Eq. (25) to Eq. (21), we finally reach

$$
I_{\mu}(t)=I_{\mu}^{\text {Steady }}\left(\alpha_{t}\right)+\sum_{n=1}^{\infty} I_{\mu}^{a(n)}(t),
$$

with $I_{\mu}^{a(n)}(t) \stackrel{\text { def }}{=}\left\langle\left\langle 1\left|W_{\mu}\left(\alpha_{t}\right)\right| \rho^{a(n)}(t)\right\rangle\right\rangle$. Since $\frac{d}{d t} \alpha_{t}=O(\omega)$ and $\mathcal{R}\left(\alpha_{t}\right)=O\left(\frac{1}{\Gamma}\right)$,

$$
\rho^{a(n)}(t)=O\left(\frac{\omega}{\Gamma}\right)^{n} .
$$

In Appendix D, we discuss the reasonable range of $n$ of $\rho^{a(n)}(t)$ and show that with the larger nonadiabaticity $\left(\frac{\omega}{\Gamma}\right)$, the reasonable range becomes wider.

Let's consider the relation between Eq. (19) and Eq. (26). In Sec. II A, we used $\chi$-adiabatic approximation Eq. (8), which becomes $\left.|\rho(t)\rangle\rangle \approx\left|\rho_{0}\left(\alpha_{t}\right)\right\rangle\right\rangle$ at $\chi=0$. Substituting it to Eq. (21), we obtain $I_{\mu}(t) \approx I_{\mu}^{\text {Steady }}(t)$. So, we cannot obtain nonadiabatic currents $\sum_{n=1}^{\infty} I_{\mu}^{a(n)}(t)$. However, from the $\chi_{\mu}$ derivative of Eq. (8), we obtain

$$
I_{\mu}(t) \approx \lambda_{0}^{\mu}\left(\alpha_{t}\right)-\left\langle\left\langle l_{0}^{\mu}\left(\alpha_{t}\right)\left|\frac{d}{d t}\right| \rho_{0}\left(\alpha_{t}\right)\right\rangle\right\rangle .
$$

This is equivalent to Eq. (10) for $\rho(0)=\rho_{0}\left(\alpha_{0}\right)$. Equation (28) suggests

$$
I_{\mu}^{a(1)}(t)=-\left\langle\left\langle l_{0}^{\mu}\left(\alpha_{t}\right)\left|\frac{d}{d t}\right| \rho_{0}\left(\alpha_{t}\right)\right\rangle\right\rangle .
$$

In fact, this is equivalent to $I_{\mu}^{a(1)}(t)=\left\langle\left\langle 1\left|W_{\mu}\left(\alpha_{t}\right)\right| \rho^{a(1)}(t)\right\rangle\right\rangle$, namely

$$
I_{\mu}^{a(1)}(t)=\left\langle\left\langle 1\left|W_{\mu}\left(\alpha_{t}\right) \mathcal{R}\left(\alpha_{t}\right) \frac{d}{d t}\right| \rho_{0}\left(\alpha_{t}\right)\right\rangle\right\rangle,
$$

because of

$$
\left\langle\langle 1| W_{\mu}(\alpha) \mathcal{R}(\alpha)=-\left\langle\left\langle l_{0}^{\mu}(\alpha)\right|+c_{\mu}(\alpha)\langle\langle 1|,\right.\right.
$$

which was shown by Sagawa et al. [47] for a single counting field. Here, $c_{\mu}(\alpha)$ are constants shown in Eq. (E7). We prove Eq. (31) at Appendix E. Equation (19) and Eq. (26) are identical because of Eq. (23) and Eq. (31). In other words, in the expansion of $I_{\mu}(t)$ obtained from a substitution of $\rho(t)=\rho_{0}\left(\alpha_{t}\right)+\sum_{n=1}^{\infty} \rho^{a(n)}(t) \equiv \sum_{n=0}^{\infty} \rho^{a(n)}(t)$ into Eq. (19), the $n$th $(n=0,1, \ldots)$ order nonadiabatic solution, $\rho^{a(n)}(t)$, gives $(n+1)$ th order nonadiabatic currents $I_{\mu}^{a(n+1)}(t)$ because of Eq. (31). Hence the FCS-QME approach picks out one higher order nonadiabatic piece of information from the solution of the QME.

Moreover, although the BSN phase [i.e., the argument of the exponential function of Eq. (6)] is derived under the $\chi$ adiabatic condition which makes Eqs. (6) and (7) appropriate, its origin is probably a nonadiabatic effect that comes from $\frac{\omega}{\Gamma}$, because Eq. (29) shows that the BSN phase has the information of the nonadiabatic part of the QME $\left[\rho^{a}(t)=\rho(t)-\rho_{0}\left(\alpha_{t}\right)\right]$. 
It is important to recognize the relations between the FCS-QME approach and the RT approach [30-36]. In the RT approach, $p_{\kappa}(t)=\langle\kappa|\rho(t)| \kappa\rangle$ are governed by the generalized master equation (GME)

$$
\frac{d}{d t} p_{\kappa}(t)=\sum_{\eta} \int_{-\infty}^{t} d t^{\prime} W_{\kappa \eta}\left(t, t^{\prime}\right) p_{\eta}\left(t^{\prime}\right)
$$

where $|\kappa\rangle$ are the energy eigenstates of the system Hamiltonian. The kernel $W_{\kappa \eta}\left(t, t^{\prime}\right)$ can include the higher order contribution of the tunneling interaction between baths and the system. In the GME, $p_{\eta}\left(t^{\prime}\right)$ is given by $p_{\eta}(t)+$ $\sum_{k=1}^{\infty} \frac{\left(t^{\prime}-t\right)^{k}}{k !} \frac{d^{k} p_{\eta}(t)}{d t^{k}}$ [30,31]. Moreover, $W_{\kappa \eta}\left(t, t^{\prime}\right)$ and $p_{\eta}(t)$ are expanded as $W_{\kappa \eta}\left(t, t^{\prime}\right)=\sum_{n=0}^{\infty} \sum_{j=1}^{\infty} W_{\kappa \eta(j)}^{(n)}\left(t ; t-t^{\prime}\right)$ and $p_{\eta}(t)=\sum_{n=0}^{\infty} \sum_{j=-n}^{\infty} p_{\eta(j)}^{(n)}(t)$, where $W_{\kappa \eta(j)}^{(n)}\left(t ; t-t^{\prime}\right)$ and $p_{\eta(j)}^{(n)}(t)$ are of the order of $\omega^{n} \Gamma^{j}$. In particular, $W_{\kappa \eta(j)}^{(0)}(t ; t-$ $\left.t^{\prime}\right)=W_{\kappa \eta(j)}^{(0)}\left(\alpha_{t} ; t-t^{\prime}\right)$ is the kernel where the control parameters are fixed to $\alpha_{t}$. Up to the second order of the tunneling interaction (in the following we consider this level of approximation), we obtain $[31,35]$

$$
\begin{gathered}
0=\sum_{\eta} K_{\kappa \eta}^{(0)}\left(\alpha_{t}\right) p_{\eta}^{(0)}\left(\alpha_{t}\right), \\
\frac{d p_{\kappa(-n)}^{(n)}(t)}{d t}=\sum_{\eta} K_{\kappa \eta}^{(0)}\left(\alpha_{t}\right) p_{\eta(-n-1)}^{(n+1)}(t),
\end{gathered}
$$

for $n=0,1, \ldots$, with

$$
K_{\kappa \eta}^{(0)}\left(\alpha_{t}\right)=\int_{-\infty}^{t} d t^{\prime} W_{\kappa \eta(1)}^{(0)}\left(\alpha_{t}, t-t^{\prime}\right),
$$

which is the instantaneous Liouvillian corresponding to our $\hat{K}\left(\alpha_{t}\right)$. Equation (33) is just the definition of the instantaneous steady state $p_{\eta}^{(0)}\left(\alpha_{t}\right) \equiv p_{\eta(0)}^{(0)}(t)$, which satisfies $\sum_{\kappa} p_{\kappa}^{(0)}\left(\alpha_{t}\right)=$ 1. Additionally, $p_{\kappa(j)}^{(n)}(t)$ for $n \geqslant 1$ satisfies $\sum_{\kappa} p_{\kappa(j)}^{(n)}(t)=0$. The conservation of the probability leads to $\sum_{\kappa} K_{\kappa \eta}^{(0)}\left(\alpha_{t}\right)=0$, which corresponds to our $\left\langle\langle 1| \hat{K}\left(\alpha_{t}\right)=0\right.$. The charge or spin current $I_{\mu}(t)$ is given by $[34,35]$

$$
I_{\mu}(t)=\sum_{\kappa, \eta} w_{\kappa \eta}^{[\mu]}\left(\alpha_{t}\right) p_{\eta}(t),
$$

corresponding to our Eq. (21). $w_{\kappa \eta}^{[\mu]}\left(\alpha_{t}\right)$ is the instantaneous current matrix of $O_{\mu}$ in the present approximation, which corresponds to our $W_{\mu}\left(\alpha_{t}\right)$ and is linear in $\Gamma$. Substituting $p_{\eta}(t) \approx \sum_{n=0}^{\infty} p_{\eta(-n)}^{(n)}(t)$ into Eq. (36), we obtain

$$
I_{\mu}(t)=\sum_{n=0}^{\infty} I_{\mu}^{(n)}(t), \quad I_{\mu}^{(n)}(t)=\sum_{\kappa, \eta} w_{\kappa \eta}^{[\mu]}\left(\alpha_{t}\right) p_{\eta(-n)}^{(n)}(t) .
$$

Equation (34) for $n=0$ leads to [34]

$$
p_{\eta(-1)}^{(1)}(t)=\sum_{\kappa} R_{\eta \kappa}\left(\alpha_{t}\right) \frac{d p_{\kappa}^{(0)}\left(\alpha_{t}\right)}{d t} .
$$

Here, $R_{\eta \kappa}\left(\alpha_{t}\right)$ is the pseudoinverse of $K_{\kappa \eta}^{(0)}\left(\alpha_{t}\right)$ corresponding to our $\mathcal{R}\left(\alpha_{t}\right)$ and it is given by [34]

$$
R_{\eta \kappa}\left(\alpha_{t}\right)=\left(\tilde{K}^{-1}\right)_{\eta \kappa}, \quad \tilde{K}_{\eta \kappa}=K_{\eta \kappa}^{(0)}-K_{\eta \eta}^{(0)} .
$$

Substituting Eq. (38) into Eq. (37), we obtain [34]

$$
\begin{aligned}
& I_{\mu}^{(1)}(t)=\sum_{\kappa} \varphi_{\kappa}^{[\mu]}\left(\alpha_{t}\right) \frac{d p_{\kappa}^{(0)}\left(\alpha_{t}\right)}{d t}, \\
& \varphi_{\kappa}^{[\mu]}\left(\alpha_{t}\right)=\sum_{\zeta, \eta} w_{\zeta \eta}^{[\mu]}\left(\alpha_{t}\right) R_{\eta \kappa}\left(\alpha_{t}\right) .
\end{aligned}
$$

A similar method has been used in Ref. [30]. $\varphi_{\kappa}^{[\mu]}\left(\alpha_{t}\right)$ and Eq. (40) respectively correspond to our $\left\langle\langle 1| W_{\mu}(\alpha) \mathcal{R}(\alpha)\right.$ and Eq. (30). Moreover, Eq. (34) for arbitrary $n$ leads to

$$
p_{\eta(-n-1)}^{(n+1)}(t)=\sum_{\kappa} R_{\eta \kappa}\left(\alpha_{t}\right) \frac{d p_{\kappa(-n)}^{(n)}(t)}{d t},
$$

which corresponds to our Eq. (25). Because of these relations, the RT approach is equivalent to the FCS-QME approach in the calculation up to the second order of the tunneling interaction. Additionally, we discuss corrections due to the nonadiabatic effect of the FCS-QME in Appendix D. The first equation of Eq. (D6) is consistent with $p_{\eta(0)}^{(1)}(t)=O\left(\omega \tau_{B}\right)$, which can be derived from Ref. [31]. Here, $\tau_{B}$ is the relaxation time of the baths.

In this section, we proved the equivalence between Eq. (19) and Eq. (26) using a key relation Eq. (31) and showed the origin of the BSN phase is a nonadiabatic effect, and connected the FCS-QME approach and the RT approach[34]. These are among the most important results of this paper.

\section{MODEL}

We consider quantum dots (QDs) (denoted by a symbol $S$ ) weakly coupled to two leads. The total Hamiltonian is $H_{\text {tot }}(t)=H_{S}(t)+\sum_{b=L, R}\left[H_{b}(t)+H_{S b}(t)\right]$. Here, $H_{S}(t)$ is the system (QDs) Hamiltonian, $H_{b}(t)$ is the Hamiltonian of lead $b=L, R$, and $H_{S b}(t)$ is the tunneling interaction Hamiltonian between $S$ and lead $b$. To observe the spin effects, we suppose that the leads and the system are applied to collinear magnetic fields with different amplitudes, which relate to spins through the Zeeman effect. The leads are noninteracting:

$$
H_{b}(t)=\sum_{k, \sigma}\left(\varepsilon_{b k}+\sigma g_{b} B_{b}(t)\right) c_{b k \sigma}^{\dagger} c_{b k \sigma} .
$$

Here, $\sigma=\uparrow, \downarrow= \pm 1$ is spin label, $g_{b}=\frac{1}{2} \mu_{\mathrm{B}} g_{b}^{*}$, where $g_{b}^{*}$ is the $g$ factor of lead $b, \mu_{\mathrm{B}}$ is the Bohr magneton, and $B_{b}(t)$ is the strength of the magnetic field of lead $b . c_{b k \sigma}^{\dagger}\left(c_{b k \sigma}\right)$ is the creation (annihilation) operator of an electron with spin $\sigma$ and momentum $k$ in lead $b$. The system Hamiltonian is

$$
H_{S}(t)=\sum_{n, m, s, s^{\prime}} \varepsilon_{n s, m s^{\prime}}\left(B_{S}(t)\right) a_{n s}^{\dagger} a_{m s^{\prime}}+H_{\text {Coulomb }},
$$

where $a_{n s}^{\dagger}$ is the creation operator of an electron with orbital $n$ and spin $s . \varepsilon_{n s, m s^{\prime}}\left(B_{S}(t)\right)$ means the energy of the electron for $n=m, s=s^{\prime}$ and the tunneling amplitude between orbitals for $(n, s) \neq\left(m, s^{\prime}\right)$, which depends on the magnetic field of the system. $H_{\text {Coulomb }}$ denotes Coulomb interaction. The tunneling interaction Hamiltonian is

$$
H_{S b}(t)=\sum_{k, \sigma, n, s} \sqrt{\Delta_{b}(t)} v_{b k \sigma, n s} a_{n s}^{\dagger} c_{b k \sigma}+\text { H.c., }
$$


where $\Delta_{b}(t)$ is a dimensionless parameter and $v_{b k \sigma, n s}$ is the tunneling amplitude.

We assume $B_{S}, B_{L / R}$, and $\Delta_{L / R}$ are control parameters (denoted $\alpha^{\prime}=\left\{B_{S}, B_{L / R}, \Delta_{L / R}\right\}$ and are called the dynamic parameters). The thermodynamic parameters (the chemical potentials and inverse temperatures of leads, $\left\{\mu_{b}\right\}$ and $\left\{\beta_{b}\right\}$ ) are also considered as control parameters in Sec. IV B and Sec. VB. We denote $\alpha^{\prime \prime}=\left\{\beta_{b}, \mu_{b}\right\}_{b=L, R}$ and $\alpha=\alpha^{\prime}+\alpha^{\prime \prime}$. Yuge et al. [43] chose the set of control parameters as only $\alpha^{\prime \prime}$. However, we are interested in $\alpha^{\prime}$ for the reason explained in Sec. IV B.

We choose the measured observables $\left\{O_{\mu}\right\}=\left\{N_{b \sigma}\right\}_{\sigma=\uparrow, \downarrow}^{b=L, R}$ with $N_{b \sigma}=\sum_{k} c_{b k \sigma}^{\dagger} c_{b k \sigma}$. The pumped charge (spin) of lead $b$ is given by $\left\langle\Delta N_{b \uparrow}\right\rangle \pm\left\langle\Delta N_{b \downarrow}\right\rangle .\left\langle\Delta N_{b \sigma}\right\rangle$ are calculated by Eq. (12). In fact, what we call the pumped charge, $\left\langle\Delta N_{b \uparrow}\right\rangle+\left\langle\Delta N_{b \downarrow}\right\rangle$, is the pumped electron number (actual pumped charge is given by $-e\left[\left\langle\Delta N_{b \uparrow}\right\rangle+\left\langle\Delta N_{b \downarrow}\right\rangle\right]$, where $e(>0)$ is the elementary charge).

In Sec. IV C and Sec. V we consider a one level system

$$
H_{S}(t)=\sum_{s=\uparrow, \downarrow} \omega_{s}\left(B_{S}(t)\right) a_{s}^{\dagger} a_{s}+U a_{\uparrow}^{\dagger} a_{\uparrow} a_{\downarrow}^{\dagger} a_{\downarrow}
$$

as a special model of Eq. (44). Here, $s=\uparrow, \downarrow= \pm 1, \omega_{s}\left(B_{S}\right)=$ $\omega_{0}+s g_{S} B_{S}$, with $\omega_{0}$ the electron energy at $B_{S}=0$, and $g_{S}=$ $\frac{1}{2} \mu_{\mathrm{B}} g_{S}^{*}$, where $g_{S}^{*}$ is the $g$ factor of the QD.

In the following, we apply the FCS-QME with rotating wave approximation (RWA) explained in Appendix B.

\section{NONINTERACTING SYSTEM}

In this section, we consider a noninteracting system $\left(H_{\text {Coulomb }}=0\right)$. The system Hamiltonian Eq. (44) can be diagonalized

$$
H_{S}=\sum_{i=1}^{2 N} \tilde{\omega}_{i} b_{i}^{\dagger} b_{i}
$$

by a unitary transform $a_{n s}=\sum_{i=1}^{2 N} U_{n s, i} b_{i}$. The tunneling interaction Hamiltonian Eq. (45) is

$$
H_{S b}=\sum_{k, \sigma, i} W_{b k \sigma, i} b_{i}^{\dagger} c_{b k \sigma}+\text { H.c., }
$$

with $W_{b k \sigma, i}=\sum_{n, s} \sqrt{\Delta_{b}} v_{b k \sigma, n s} U_{n s, i}^{*}$.

In Sec. IV A, the Liouvillian and its instantaneous steady state are explained. In Sec. IV B, we consider the contribution of Eq. (13) and show that this cannot be neglected in general if the chemical potentials and the temperatures are not fixed. In Sec. IV C, we calculate the BSN curvatures for two combinations of modulated control parameters $\left(B_{L}, B_{S}\right)$ and $\left(\Delta_{L}, B_{S}\right)$.

\section{A. Liouvillian}

The Liouvillian in the RWA is given by

$$
\begin{gathered}
\hat{K}^{\chi}(\alpha)=\sum_{i=1}^{2 N} \hat{K}_{i}^{\chi}(\alpha), \\
\hat{K}_{i}^{\chi}(\alpha) \bullet=-i\left[\tilde{\omega}_{i} b_{i}^{\dagger} b_{i}, \bullet\right]+\hat{\Pi}_{i}^{\Phi}(\chi, \alpha) \bullet+\hat{\Pi}_{i}^{\Psi}(\alpha) \bullet,
\end{gathered}
$$

if $\left\{\tilde{\omega}_{i}\right\}$ are not degenerated. Here, superoperators $\hat{\Pi}_{i}^{\Phi}(\chi, \alpha)$ and $\hat{\Pi}_{i}^{\Psi}(\alpha)$ operate to an arbitrary operator $\bullet$ as

$$
\begin{gathered}
\hat{\Pi}_{i}^{\Phi}(\chi, \alpha) \bullet \\
=\sum_{b}\left\{\Phi_{b, i}^{+, \chi} b_{i}^{\dagger} \bullet b_{i}-\frac{1}{2} \Phi_{b, i}^{+} \bullet b_{i} b_{i}^{\dagger}-\frac{1}{2} \Phi_{b, i}^{+} b_{i} b_{i}^{\dagger} \bullet\right. \\
\left.+\Phi_{b, i}^{-, \chi} b_{i} \bullet b_{i}^{\dagger}-\frac{1}{2} \Phi_{b, i}^{-} \bullet b_{i}^{\dagger} b_{i}-\frac{1}{2} \Phi_{b, i}^{-} b_{i}^{\dagger} b_{i} \bullet\right\}, \\
\hat{\Pi}_{i}^{\Psi}(\alpha) \bullet=i\left[\Omega_{i}(\alpha) b_{i}^{\dagger} b_{i}, \bullet\right],
\end{gathered}
$$

with

$$
\begin{gathered}
\Phi_{b, i}^{ \pm, \chi}=2 \pi \sum_{k, \sigma}\left|W_{b k \sigma, i}\right|^{2} f_{b}^{ \pm}\left(\tilde{\omega}_{i}\right) e^{\mp i \chi \chi_{b \sigma}} \\
\times \delta\left(\varepsilon_{b k}+\sigma g_{b} B_{b}-\tilde{\omega}_{i}\right), \\
\Psi_{b, i}^{ \pm}=2 \sum_{k, \sigma}\left|W_{b k \sigma, i}\right|^{2} f_{b}^{ \pm}\left(\tilde{\omega}_{i}\right) \mathrm{P} \frac{1}{\varepsilon_{b k}+\sigma g_{b} B_{b}-\tilde{\omega}_{i}},
\end{gathered}
$$

and $\Omega_{i}(\alpha)=\frac{1}{2} \sum_{b}\left(\Psi_{b, i}^{-}+\Psi_{b, i}^{+}\right)$. Here, $f_{b}^{+}(\omega)=\left[e^{\beta_{b}\left(\omega-\mu_{b}\right)}+\right.$ $1]^{-1}$ is the Fermi distribution function, $f_{b}^{-}(\omega)=1-f_{b}^{+}(\omega)$, $\chi_{b \sigma}$ is the counting field for $N_{b \sigma}$, and P denotes the Cauchy principal value. The matrix representation of $\hat{K}_{i}^{\chi}(\alpha)$ (see Appendix A) by the number states of $b_{i}^{\dagger} b_{i}\left(|0\rangle_{i}\right.$ and $\left.|1\rangle_{i}\right)$ is a $4 \times 4$ matrix which is block diagonalized to $\left\{|0\rangle_{i i}\langle 0|,| 1\rangle_{i i}\langle 1|\right\}$ space and $\left\{|0\rangle_{i i}\langle 1|,| 1\rangle_{i i}\langle 0|\right\}$ space. The $\left\{|0\rangle_{i i}\langle 0|,| 1\rangle_{i i}\langle 1|\right\}$ part is given by

$$
\left.\left.K_{i}^{\chi}(\alpha)=\left(\begin{array}{cc}
-\Phi_{i}^{+} & \Phi_{i}^{-, \chi} \\
\Phi_{i}^{+, \chi} & -\Phi_{i}^{-}
\end{array}\right)|00\rangle\right\rangle_{i},|11\rangle\right\rangle_{i},
$$

with $\Phi_{i}^{ \pm, \chi}=\sum_{b} \Phi_{b, i}^{ \pm, \chi} .\left\{|0\rangle_{i i}\langle 1|,| 1\rangle_{i i}\langle 0|\right\}$ part does not relate to the instantaneous steady state of $\hat{K}_{i}^{\chi}(\alpha)$. The eigenvalue of the instantaneous steady state of $\hat{K}_{i}^{\chi}(\alpha)$ is given by

$$
\lambda_{i, 0}^{\chi}(\alpha)=-\frac{\Phi_{i}^{+}(\alpha)+\Phi_{i}^{-}(\alpha)}{2}+\sqrt{D_{i}^{\chi}(\alpha)},
$$

with $D_{i}^{\chi}(\alpha)=\left[\Phi_{i}^{+}+\Phi_{i}^{-}\right]^{2} / 4-\left[\Phi_{i}^{+} \Phi_{i}^{-}-\Phi_{i}^{-, \chi} \Phi_{i}^{+, \chi}\right]$. The corresponding left and right eigenvectors are $\left.\left|\rho_{i, 0}^{\chi}(\alpha)\right\rangle\right\rangle$ $\left.\left.=C_{i}^{\chi}(\alpha)|00\rangle\right\rangle_{i}+E_{i}^{\chi}(\alpha)|11\rangle\right\rangle_{i} \quad$ and $\quad\left\langle\left\langle l_{0, i}^{\chi}(\alpha)\right|={ }_{i}\langle\langle 00|+\right.$ $v_{i}^{\chi}(\alpha)_{i}\left\langle\langle 11| \quad\right.$ with $\quad C_{i}^{\chi}(\alpha)=\frac{\Phi_{i}^{-, \chi} \Phi_{i}^{+, \chi}}{\left[\lambda_{i, 0}^{\chi}+\Phi_{i}^{+}\right]^{2}+\Phi_{i}^{-, \chi} \Phi_{i}^{+, \chi}}, \quad E_{i}^{\chi}(\alpha)=$ $\frac{\Phi_{i}^{+, \chi}\left(\lambda_{i, 0}^{\chi}+\Phi_{i}^{+}\right)}{\left[\lambda_{i, 0}^{\chi}+\Phi_{i}^{+}\right]^{2}+\Phi_{i}^{-, \chi \chi} \Phi_{i}^{+, \chi}}$, and

$$
v_{i}^{\chi}(\alpha)=\frac{\Phi_{i}^{+}-\Phi_{i}^{-}+2 \sqrt{D_{i}^{\chi}(\alpha)}}{2 \Phi_{i}^{+, \chi}} .
$$

At $\chi_{b \sigma}=0, E_{i}^{\chi}(\alpha)$ becomes $E_{i}(\alpha)=\frac{\Phi_{i}^{+}}{\Phi_{i}^{+}+\Phi_{i}^{-}}$and $C_{i}^{\chi}(\alpha)$ becomes $C_{i}(\alpha)=1-E_{i}(\alpha)$.

\section{B. Instantaneous steady currents}

The instantaneous steady current is given by $I_{b \sigma}^{\text {Steady }}(\alpha)=$ $\left.\frac{\partial \lambda_{0}^{\chi}(\alpha)}{\partial\left(i \chi_{b \sigma}\right)}\right|_{\chi=0}$. In the noninteracting system, $\lambda_{0}^{\chi}(\alpha)$ is $\sum_{i} \lambda_{i, 0}^{\chi}(\alpha)$ and it leads to $I_{b \sigma}^{\text {Steady }}(\alpha)=\sum_{i} I_{i, b \sigma}^{\text {Steady }}(\alpha)$. Here, $I_{i, b \sigma}^{\text {Steady }}(\alpha)=$ 
$\left.\frac{\partial \lambda_{0, i}^{\chi}(\alpha)}{\partial\left(i \chi_{b \sigma}\right)}\right|_{\chi=0}$ are calculated from Eq. (56) as

$$
I_{i, L \sigma}^{\text {Steady }}(\alpha)=\frac{\Gamma_{L \sigma, i} \Gamma_{R, i}\left(f_{R}\left(\tilde{\omega}_{i}\right)-f_{L}\left(\tilde{\omega}_{i}\right)\right)}{\Gamma_{i}},
$$

with $\quad \Gamma_{b \sigma, i}=2 \pi \sum_{k}\left|W_{b k \sigma, i}\right|^{2} \delta\left(\varepsilon_{b k}+\sigma g_{b} B_{b}-\tilde{\omega}_{i}\right), \quad \Gamma_{b, i}=$ $\sum_{\sigma} \Gamma_{b \sigma, i}$, and $\Gamma_{i}=\sum_{b} \Gamma_{b, i} . I_{i, L \sigma}^{\text {Steady }}(\alpha)$ vanishes at zero bias $\left(\beta_{L}=\beta_{R}, \mu_{L}=\mu_{R}\right)$. Let us consider the modulation of only the thermodynamic parameters $\left(\alpha^{\prime \prime}=\left\{\mu_{b}, \beta_{b}\right\}_{b=L, R}\right)$ similar to Refs. [43,52,53,57]. The factor depending on $\alpha^{\prime \prime}$ of $I_{i, b \sigma}^{\text {Steady }}\left(\alpha_{t}\right)$ is $\left[f_{\beta_{R}(t), \mu_{R}(t)}\left(\tilde{\omega}_{i}\right)-f_{\beta_{L}(t), \mu_{L}(t)}\left(\tilde{\omega}_{i}\right)\right]$ with $f_{\beta, \mu}(\omega)=$ $\left[e^{\beta(\omega-\mu)}+1\right]^{-1}$. Hence

$$
\begin{aligned}
\left\langle\Delta N_{b \sigma}\right\rangle_{\tau}^{\text {Steady }}= & \sum_{i} \frac{\Gamma_{L \sigma, i} \Gamma_{R, i}}{\Gamma_{i}} \\
& \times \int_{0}^{\tau} d t\left[f_{\beta_{R}(t), \mu_{R}(t)}\left(\tilde{\omega}_{i}\right)-f_{\beta_{L}(t), \mu_{L}(t)}\left(\tilde{\omega}_{i}\right)\right]
\end{aligned}
$$

is generally nonzero and is much lager than $\left\langle\Delta N_{b \sigma}\right\rangle_{S}^{\text {Berry }}$ because the period $\tau$ is large for adiabatic pumps. Similarly, we can show that $\left\langle\Delta N_{b \sigma}\right\rangle_{\tau}^{\text {Steady }}$ is generally nonzero for the interacting system (Sec. VB). Reference [53] considered special modulations of only thermodynamic parameters which satisfy $\left\langle\Delta N_{b \sigma}\right\rangle_{\tau}^{\text {Steady }}=0$. In fact, the instantaneous steady currents are always zero for arbitrary modulations of only the dynamics parameters at zero bias.

The pumped charge and spin due to the instantaneous steady currents (backgrounds) are generally nonzero even if the time averages of the bias are zero. References $[33,34]$ chose $V=$ $\mu_{L}-\mu_{R}$ as one of the modulating parameters and considered a pumping such that $\frac{1}{\tau} \int_{0}^{\tau} d t V(t)=0$ and $\left\langle\Delta N_{b \sigma}\right\rangle_{\tau}^{\text {Steady }} \neq 0$. In such pumping, the (thermal or voltage) bias is effectively nonzero.

Even if the backgrounds do not vanish, one can detect the BSN curvatures by subtracting the backgrounds by using zero-frequency measurements or by lock-in measurements. However, if one wants to apply the adiabatic pump to the current standard [12,13], the instantaneous steady currents should be zero at all times because the backgrounds are sensitive to the velocity of the modulation of the control parameters and its trajectory. In contrast, the pumped charge and spin due to the BSN curvatures are robust against the modulation of the velocity and the trajectory. Hence, if one wants to directly apply the BSN curvatures to, for instance, the current standard, one should fix the thermodynamic parameters at zero bias.

\section{BSN curvatures}

In the following, we consider one level system of which the Hamiltonian is Eq. (46) at $U=0$. The instantaneous steady state is given by $\left.\left.\left|\rho_{0}^{\chi}(\alpha)\right\rangle\right\rangle=\otimes_{s=\uparrow, \downarrow}\left|\rho_{s, 0}^{\chi}(\alpha)\right\rangle\right\rangle$ because the Liouvillian is described by a summation $\left(\hat{K}^{\chi}=\sum_{s=\uparrow, \downarrow} \hat{K}_{s}^{\chi}\right)$. Similarly, the corresponding left eigenvalue is given by $\left\langle\left\langle l_{0}^{\chi}(\alpha)\right|=\otimes_{s=\uparrow, \downarrow}\left\langle\left\langle l_{s, 0}^{\chi}(\alpha)\right|\right.\right.$. The BSN vectors Eq. (11) are given by

$$
A_{n}^{b \sigma}(\alpha)=\sum_{s=\uparrow, \downarrow} v_{s}^{b \sigma}\left(\alpha^{\prime}\right) \frac{\partial E_{s}(\alpha)}{\partial \alpha^{n}}
$$

where

$$
v_{s}^{b \sigma}\left(\alpha^{\prime}\right)=\left.\frac{\partial v_{s}^{\chi}(\alpha)}{\partial\left(i \chi_{b \sigma}\right)}\right|_{\chi=0}=\frac{\Gamma_{b \sigma, s}}{\Gamma_{s}},
$$

with

$$
\begin{aligned}
\Gamma_{b \sigma, s}\left(\alpha^{\prime}\right)= & 2 \pi \Delta_{b} \sum_{k}\left|v_{b k \sigma, s}\right|^{2} \\
& \times \delta\left(\varepsilon_{b k}+\sigma g_{b} B_{b}-\omega_{0}-s g_{S} B_{S}\right) .
\end{aligned}
$$

$v_{s}^{b \sigma}\left(\alpha^{\prime}\right)$ does not depend on $\alpha^{\prime \prime}$. Equation (60) leads to an expression of the BSN curvatures

$$
F_{m n}^{b \sigma}(\alpha)=\sum_{s=\uparrow, \downarrow}\left[\frac{\partial v_{s}^{b \sigma}\left(\alpha^{\prime}\right)}{\partial \alpha^{m}} \frac{\partial E_{s}(\alpha)}{\partial \alpha^{n}}-(m \leftrightarrow n)\right]
$$

We emphasize that Eq. (63) is consistent with the results of Refs. [33,34,43], which showed that the pumped charge (and also spin in Ref. [34]) vanishes at the noninteracting limit in these settings. The set of control parameters $\alpha$ was $\alpha^{\prime \prime}$ (for Ref. [43]) and $\left\{\omega_{0}, V=\mu_{L}-\mu_{R}\right\}$ (for Refs. [33,34]). If $\alpha^{m}$ or $\alpha^{n}$ is an element of $\alpha^{\prime \prime}, F_{m n}^{b \sigma}(\alpha)$ is consistently zero. In Refs. [33,34], the linewidth functions were energy independent, namely $\Gamma_{b \sigma, s}\left(\alpha^{\prime}\right)=\delta_{\sigma, s} \Gamma_{b}=$ const. Hence $\frac{\partial \Gamma_{b \sigma, s}\left(\alpha^{\prime}\right)}{\partial \omega_{0}}=0=\frac{\partial \Gamma_{b \sigma, s}\left(\alpha^{\prime}\right)}{\partial V}$ and $F_{\omega_{0}, V}^{b \sigma}(\alpha)=0$ hold consistently.

To calculate $F_{m n}^{b \sigma}(\alpha)$, we need to assume the energy dependences of $\Gamma_{b \sigma, s}$. For the simplicity, we assume that

$$
\begin{aligned}
\Gamma_{b \sigma, s} & =\delta_{\sigma, s}\left[\Gamma_{b}+\Gamma_{b}^{\prime} \cdot\left(s g_{S} B_{S}-\sigma g_{b} B_{b}\right)\right] \\
& =\delta_{\sigma, s} \Delta_{b}\left[\gamma_{b}+\gamma_{b}^{\prime} \cdot\left(s g_{S} B_{S}-\sigma g_{b} B_{b}\right)\right],
\end{aligned}
$$

where $\Gamma_{b}^{\prime}$ are energy differential coefficients of linewidth functions at $B_{b}=B_{S}=0$. Namely, we disregard spin flips induced by tunneling between the QD and the leads. Equation (64) is always appropriate when $\mid \Gamma_{b}^{\prime}\left(g_{S} B_{S}-\right.$ $\left.g_{b} B_{b}\right) \mid \ll \Gamma_{b}$ is satisfied. Additionally, we fix $\alpha^{\prime \prime}$ to zero bias $\left(\beta_{b}=\beta, \mu_{b}=\mu\right)$, in which $E_{s}(\alpha)$ is given by $E_{s}(\alpha)=$ $f\left(\omega_{0}+s g_{S} B_{S}\right)$ with $f(\omega)=\left[e^{\beta(\omega-\mu)}+1\right]^{-1}$. In this condition, $\left(\alpha^{m}, \alpha^{n}\right)=\left(B_{L}, B_{S}\right),\left(\Delta_{L}, B_{S}\right)$ components of the charge and spin BSN curvatures of lead $L$ are

$$
\begin{aligned}
F_{B_{L}, B_{S}}^{L \uparrow} \pm F_{B_{L}, B_{S}}^{L \downarrow}= & -g_{S} g_{L} \Gamma_{L}^{\prime}\left[f^{\prime}\left(\omega_{0}+g_{S} B_{S}\right) \pm f^{\prime}\left(\omega_{0}-g_{S} B_{S}\right)\right] \frac{\Gamma_{R}}{\Gamma_{\text {tot }}^{2}}+g_{S} g_{L} \Gamma_{L}^{\prime}\left[f^{\prime}\left(\omega_{0}+g_{S} B_{S}\right) \mp f^{\prime}\left(\omega_{0}-g_{S} B_{S}\right)\right] \\
& \times\left(\Gamma_{L}^{\prime}\left(g_{S} B_{S}-g_{L} B_{L}\right) \frac{2 \Gamma_{R}}{\Gamma_{\text {tot }}^{3}}+\Gamma_{R}^{\prime}\left(g_{S} B_{S}-g_{R} B_{R}\right) \frac{\Gamma_{R}-\Gamma_{L}}{\Gamma_{\text {tot }}^{3}}\right),
\end{aligned}
$$




$$
\begin{aligned}
F_{\Delta_{L}, B_{S}}^{L \uparrow} \pm F_{\Delta_{L}, B_{S}}^{L \downarrow}= & g_{S}\left[f^{\prime}\left(\omega_{0}+g_{S} B_{S}\right) \mp f^{\prime}\left(\omega_{0}-g_{S} B_{S}\right)\right] \frac{\gamma_{L} \gamma_{R} \Delta_{R}}{\left(\gamma_{L} \Delta_{L}+\gamma_{R} \Delta_{R}\right)^{2}} \\
& +g_{S}\left[f^{\prime}\left(\omega_{0}+g_{S} B_{S}\right) \pm f^{\prime}\left(\omega_{0}-g_{S} B_{S}\right)\right] \gamma_{L}^{\prime}\left(g_{S} B_{S}-g_{L} B_{L}\right) \frac{\gamma_{R} \Delta_{R}-\gamma_{L} \Delta_{L}}{\left(\gamma_{L} \Delta_{L}+\gamma_{R} \Delta_{R}\right)^{2}}
\end{aligned}
$$

Here $f^{\prime}(\omega)=\frac{\partial f(\omega)}{\partial \omega}$ and $\Gamma_{\text {tot }}=\Gamma_{L}+\Gamma_{R}$. The pumped charge (spin) induced by a slow cycle modulation of $\left(\alpha^{n}, B_{S}\right)\left(\alpha^{n}=\right.$ $\left.B_{L}, \Delta_{L}\right)$ are given by

$$
\left\langle\Delta N_{L \uparrow}\right\rangle \pm\left\langle\Delta N_{L \downarrow}\right\rangle=-\int_{S^{n}} d \alpha^{n} d B_{S}\left(F_{\alpha^{n}, B_{S}}^{L \uparrow} \pm F_{\alpha^{n}, B_{S}}^{L \downarrow}\right),
$$

where $S^{n}$ are areas enclosed by the trajectories of $\left(\alpha^{n}, B_{S}\right)$. $F_{\alpha^{n}, B_{S}}^{L \uparrow} \pm F_{\alpha^{n}, B_{S}}^{L \downarrow}\left(\alpha^{n}=B_{L}, \Delta_{L}\right)$ are invariant under the transformation $\gamma_{b} \rightarrow c \gamma, \gamma_{b}^{\prime} \rightarrow c \gamma_{b}^{\prime}$ (for any $c>0$ ). Hence relevant quantities are $\gamma_{b}^{\prime} / \Gamma_{\text {tot }}$. The coupling strength $\Gamma_{\text {tot }}$ itself is not important. $F_{B_{L}, B_{S}}^{L \uparrow} \pm F_{B_{L}, B_{S}}^{L \downarrow}$ are proportional to $g_{S} g_{L}$ and $F_{\Delta_{L}, B_{S}}^{L \uparrow} \pm F_{\Delta_{L}, B_{S}}^{L \downarrow}$ are proportional to $g_{S}$. The first terms of the right side of Eq. (65) and Eq. (66) are dominant terms. In the limit $\gamma_{L}^{\prime} \rightarrow 0, F_{B_{L}, B_{S}}^{L \uparrow} \pm F_{B_{L}, B_{S}}^{L \downarrow}$ and the second term of Eq. (66) vanish; however, the dominant term of Eq. (66) remains. At $\omega_{0}=\mu, f^{\prime}\left(\omega_{0}+g_{S} B_{S}\right)-f^{\prime}\left(\omega_{0}-g_{S} B_{S}\right)$ vanish. Hence, at $\omega_{0}=\mu$, the dominant terms of the spin BSN curvature of $\left(B_{L}, B_{S}\right)$ pump and the charge BSN of $\left(\Delta_{L}, B_{S}\right)$ pump vanish. The contour plots of these BSN curvatures are shown in Figs. 1(a) and 1(b) and Figs. 2(a) and 2(b). The details are explained in Sec. V C.

It is important to remark that $\left(\alpha^{m}, \alpha^{n}\right)=\left(B_{L}, B_{R}\right),\left(\Delta_{L}, \Delta_{R}\right)$ components of the charge and spin BSN curvatures are zero

$$
K^{\chi}(\alpha)=\left(\begin{array}{cc}
-\left[\Phi_{\uparrow}^{+}+\Phi_{\downarrow}^{+}\right] & \Phi_{\uparrow}^{-, \chi} \\
\Phi_{\uparrow}^{+, \chi} & -\left[\Phi_{\uparrow}^{-}+\phi_{\downarrow}^{+}\right] \\
\Phi_{\downarrow}^{+, \chi} & 0 \\
0 & \phi_{\downarrow}^{+, \chi}
\end{array}\right.
$$

with

$$
\begin{aligned}
\phi_{b, s}^{ \pm, \chi}= & 2 \pi \Delta_{b} \sum_{k, \sigma}\left|v_{b k \sigma, s}\right|^{2} f_{b}^{ \pm}\left(\omega_{0}+s g_{S} B_{S}+U\right) \\
& \times e^{\mp i \chi_{b \sigma}} \delta\left(\varepsilon_{b k}+\sigma g_{b} B_{b}-\omega_{0}-s g_{S} B_{S}-U\right),
\end{aligned}
$$

and $\Phi_{b, s}^{ \pm, \chi}=\left.\phi_{b, s}^{ \pm, \chi}\right|_{U=0}$. The off-diagonal block is a $(12 \times 12)$ diagonal matrix, which dose not relate to the instantaneous steady state. At $U=0, K^{\chi}(\alpha)$ becomes $K_{\uparrow}^{\chi}(\alpha) \otimes 1_{\downarrow}+1_{\uparrow} \otimes$ $K_{\downarrow}^{\chi}(\alpha)$, where $K_{s}^{\chi}(\alpha)(s=\uparrow, \downarrow)$ are given by Eq. (55) and $1_{s}$ are identity matrices. In the opposite limit $U \rightarrow \infty, K^{\chi}(\alpha)$ reduces to

$$
K^{\chi(\infty)}(\alpha)=\left(\begin{array}{ccc}
-\left[\Phi_{\uparrow}^{+}+\Phi_{\downarrow}^{+}\right] & \Phi_{\uparrow}^{-, \chi} & \Phi_{\downarrow}^{-, \chi} \\
\Phi_{\uparrow}^{+, \chi} & -\Phi_{\uparrow}^{-} & 0 \\
\Phi_{\downarrow}^{+, \chi} & 0 & -\Phi_{\downarrow}^{-}
\end{array}\right) \mid \begin{aligned}
& |0000\rangle\rangle \\
& |01010\rangle\rangle,
\end{aligned}
$$

because the density of state of both leads vanishes at high energy $\left(\phi_{s}^{ \pm} \rightarrow 0\right)$. at zero bias because, in Eq. (63), $E_{S}(\alpha)=f\left(\omega_{0}+s g_{S} B_{S}\right)$ are independent of $B_{L / R}$ and $\Delta_{L / R}$.

\section{INTERACTING SYSTEM}

In this section, we study the interacting system Eq. (46). First, we explain the Liouvillian for $0 \leqslant U \leqslant \infty$ (Sec. V A). Next, the instantaneous steady charge and spin currents are calculated at $U=\infty$ (Sec. V B). In Sec. V C, we confirm the consistency between our results and Ref. [34] for $0 \leqslant U \leqslant \infty$. Finally, the BSN curvatures corresponding to Eq. (65) and Eq. (66) are calculated at $U=\infty$ and differences of the results between $U=0$ and $U=\infty$ are discussed.

\section{A. Liouvillian}

We explain the Liouvillian for $k_{\mathrm{B}} T>\Gamma$, in which the BornMarkov approximation is appropriate. The matrix representation of the Liouvillian of RWA by the number states $\left\{\left|n_{\uparrow} n_{\downarrow}\right\rangle\right\}$ $\left(n_{s}=0,1\right.$ are the numbers of an electron with spin $\left.s=\uparrow, \downarrow\right)$ is a $16 \times 16$ matrix which is block diagonalized to the "diagonal" space (spanned by $\left\{\left|n_{\uparrow} n_{\downarrow}\right\rangle\left\langle n_{\uparrow} n_{\downarrow}\right|\right\}_{n_{\uparrow}, n_{\downarrow}=0,1}$ ) and the "offdiagonal" space (spanned by $\left\{\left|n_{\uparrow} n_{\downarrow}\right\rangle\left\langle m_{\uparrow} m_{\downarrow}\right|\right\}_{\left(n_{\uparrow}, n_{\downarrow}\right) \neq\left(m_{\uparrow}, m_{\downarrow}\right)}$ ). The "diagonal" block is given by

\begin{tabular}{cc|c}
$\Phi_{\downarrow}^{-, \chi}$ & 0 \\
0 & $\phi_{\downarrow}^{-, \chi}$ & $|0000\rangle\rangle$ \\
$-\left[\Phi_{\downarrow}^{-}+\phi_{\uparrow}^{+}\right]$ & $\phi_{\uparrow}^{-, \chi}$ & $|01010\rangle\rangle$ \\
$\phi_{\uparrow}^{+, \chi}$ & $-\left[\phi_{\uparrow}^{-}+\phi_{\downarrow}^{-}\right]$ & $|1111\rangle\rangle\rangle$
\end{tabular}

\section{B. Instantaneous steady currents}

In this section, we set $U=\infty$. The characteristic polynomial of $K^{\chi(\infty)}$ is denoted as $C_{3}(\chi, \lambda)=\operatorname{det}\left(K^{\chi(\infty)}-\right.$ $\lambda)=\sum_{n=0}^{2} C_{n}(\chi) \lambda^{n}-\lambda^{3}$. Because of $C_{0}(0)=0, \lambda=0$ is one of the solutions at $\chi=0$. Now we set $\chi_{b \sigma}$ as infinitesimal and other counting fields are zero. Then, the eigenvalue corresponding to the instantaneous steady state is given by $\lambda=\lambda_{0}(\chi)=i \chi_{b \sigma} \cdot I_{b \sigma}^{\text {Steady }}+O\left(\chi_{b \sigma}^{2}\right)$. It leads to $0=C_{3}\left(\chi, \lambda_{0}(\chi)\right)=C_{1}(0) i \chi_{b \sigma} I_{b \sigma}^{\text {Steady }}+i \chi_{b \sigma} C_{0}^{b \sigma}$ with $C_{0}^{b \sigma}=$ $\left.\frac{\partial C_{0}(\chi)}{\partial\left(i \chi_{b \sigma}\right)}\right|_{\chi=0}$, and we obtain

$$
I_{b \sigma}^{\text {Steady }}=-\frac{C_{0}^{b \sigma}}{C_{1}(0)},
$$

with $C_{1}(0)=-\left[\Phi_{\uparrow}^{+} \Phi_{\downarrow}^{-}+\Phi_{\uparrow}^{-} \Phi_{\downarrow}^{+}+\Phi_{\uparrow}^{-} \Phi_{\downarrow}^{-}\right]$. From $C_{0}(\chi)=$ $-\left[\Phi_{\uparrow}^{+}+\Phi_{\downarrow}^{+}\right] \Phi_{\uparrow}^{-} \Phi_{\downarrow}^{-}+\Phi_{\downarrow}^{-, \chi} \Phi_{\uparrow}^{-} \Phi_{\downarrow}^{+, \chi}+\Phi_{\downarrow}^{-} \Phi_{\uparrow}^{-, \chi} \Phi_{\uparrow}^{+, \chi}$, we have

$$
I_{L \sigma}^{\text {Steady }}(\alpha)=\frac{\sum_{s=\uparrow, \downarrow} \Phi_{-s}^{-} \Gamma_{L \sigma, s} \Gamma_{R, s}\left(f_{R, s}-f_{L, s}\right)}{\Phi_{\uparrow}^{+} \Phi_{\downarrow}^{-}+\Phi_{\uparrow}^{-} \Phi_{\downarrow}^{+}+\Phi_{\uparrow}^{-} \Phi_{\downarrow}^{-}},
$$



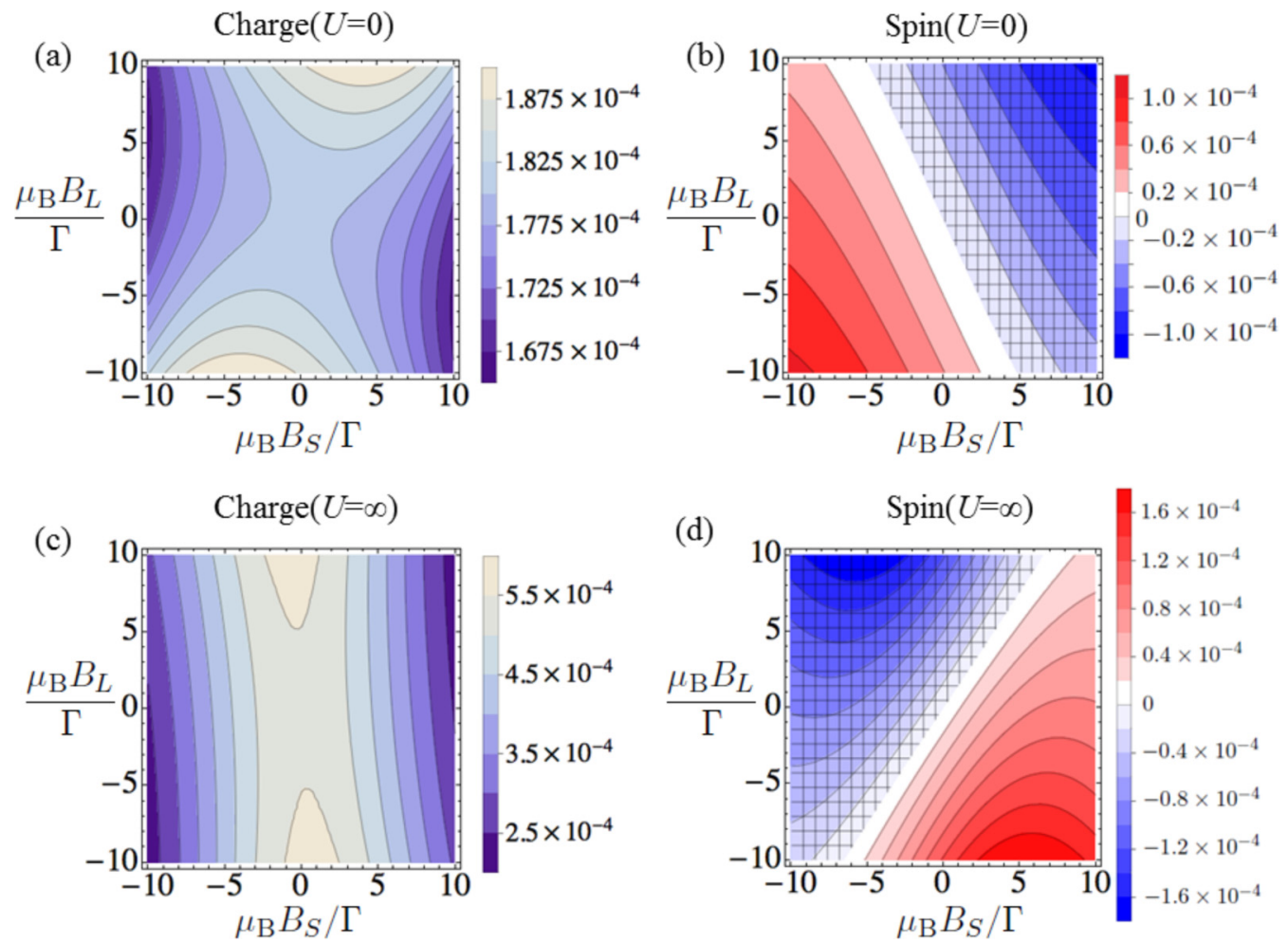

FIG. 1. (Color online) (a) BSN curvature of charge of $\left(B_{L}, B_{S}\right)$ pump, $\left[F_{B_{L}, B_{S}}^{L \uparrow}+F_{B_{L}, B_{S}}^{L \downarrow}\right] /\left(\frac{\mu_{\mathrm{B}}}{\Gamma}\right)^{2}$ at $U=0$, (b) the BSN curvature of spin, $\left[F_{B_{L}, B_{S}}^{L \uparrow}-F_{B_{L}, B_{S}}^{L \downarrow}\right] /\left(\frac{\mu_{\mathrm{B}}}{\Gamma}\right)^{2}$ at $U=0$, (c) $\left[F_{B_{L}, B_{S}}^{L \uparrow}+F_{B_{L}, B_{S}}^{L \downarrow}\right] /\left(\frac{\mu_{\mathrm{B}}}{\Gamma}\right)^{2}$ at $U=\infty$, and (d) $\left[F_{B_{L}, B_{S}}^{L \uparrow}-F_{B_{L}, B_{S}}^{L \downarrow}\right] /\left(\frac{\mu_{\mathrm{B}}}{\Gamma}\right)^{2}$ at $U=\infty$. The values of the parameters used for these plots are $\Gamma_{L}=\Gamma_{R}=\Gamma, \Gamma_{L}^{\prime}=\Gamma_{R}^{\prime}=0.1, \beta=0.5 / \Gamma, \omega_{0}=\mu-3 \Gamma$, and $B_{R}=0$, and all $g$ factors $\left(g_{L}^{*}, g_{R}^{*}, g_{S}^{*}\right)$ are -0.44 (bulk GaAs). The hatched areas of (b),(d) denote negative value.

where $\Phi_{-s}^{-}(s=\uparrow, \downarrow)$ describes $\Phi_{\downarrow}^{-}$for $s=\uparrow$ and $\Phi_{\downarrow}^{-}$for $s=\downarrow$. At zero bias, the instantaneous steady currents vanish. Similar to Sec. IV B, $\left\langle\Delta N_{b \sigma}\right\rangle_{\tau}^{\text {Steady }}$ are generally nonzero when $\alpha^{\prime \prime}$ is not fixed at zero bias.

\section{BSN curvatures}

The instantaneous steady state $\rho_{0}(\alpha)$ and corresponding left eigenvector $l_{0}^{\chi}(\alpha)$ are written as $\rho_{0}=\rho_{0}|00\rangle\langle 00|+$ $\rho_{\uparrow}|10\rangle\left\langle 10\left|+\rho_{\downarrow}\right| 01\right\rangle\left\langle 01\left|+\rho_{2}\right| 11\right\rangle\langle 11|$ and $l_{0}^{\chi}=|00\rangle\langle 00|+$ $l_{\uparrow}^{\chi}|10\rangle\left\langle 10\left|+l_{\downarrow}^{\chi}\right| 01\right\rangle\left\langle 01\left|+l_{2}^{\chi}\right| 11\right\rangle\langle 11|$. The BSN vectors are given by

$$
A_{n}^{b \sigma}(\alpha)=\sum_{c=\uparrow, \downarrow, 2} l_{c}^{b \sigma}(\alpha) \frac{\partial \rho_{c}(\alpha)}{\partial \alpha^{n}},
$$

where $l_{c}^{b \sigma}(\alpha)=\left.\frac{\partial\left[l_{c}^{\chi}(\alpha)\right]^{*}}{\partial\left(i \chi_{b \sigma}\right)}\right|_{\chi=0}$. It leads to the BSN curvatures

$$
F_{m n}^{b \sigma}(\alpha)=\sum_{c=\uparrow, \downarrow, 2} \frac{\partial l_{c}^{b \sigma}(\alpha)}{\partial \alpha^{m}} \frac{\partial \rho_{c}(\alpha)}{\partial \alpha^{n}}-(m \leftrightarrow n) .
$$

We confirmed the consistency between our results and Ref. [34], which studied the similar system for $0 \leqslant U \leqslant \infty$ using the wideband limit. As we explained at Sec. II B, $\varphi_{k}^{[\mu]}(\alpha)$ of Eq. (41) corresponds to $-\left\langle\left\langle l_{0}^{\mu}(\alpha)\right|\right.$, namely $-l_{c}^{b \sigma}(\alpha)$. In the condition of the wideband limit [i.e., Eq. (64) with $\Gamma_{b}^{\prime}=0$ ], we calculated $l_{c}^{b \sigma}(\alpha)(c=\uparrow, \downarrow, 2)$ for $0 \leqslant U \leqslant \infty$ and confirmed numerically the correspondence between $\varphi_{c}^{[\mu]}(\alpha)(c=\uparrow, \downarrow, 2)$ and $-\left[l_{c}^{b \uparrow}(\alpha) \pm l_{c}^{b \downarrow}(\alpha)\right]$ for the charge and spin pump.
Particularly, in the limit $U \rightarrow \infty, \rho_{2}$ vanishes and $F_{m n}^{b \sigma}(\alpha)$ reduces to

$$
F_{m n}^{b \sigma(\infty)}(\alpha)=\sum_{s=\uparrow, \downarrow} \frac{\partial l_{s}^{b \sigma(\infty)}(\alpha)}{\partial \alpha^{m}} \frac{\partial \rho_{s}^{(\infty)}(\alpha)}{\partial \alpha^{n}}-(m \leftrightarrow n),
$$

where $\rho_{s}^{(\infty)}(\alpha)$ and $l_{s}^{b \sigma(\infty)}(\alpha)$ are the limits $U \rightarrow \infty$ of $\rho_{s}(\alpha)$ and $l_{s}^{b \sigma}(\alpha)$, respectively. From Eq. (70) we obtain

$$
\begin{gathered}
\rho_{s}^{(\infty)}(\alpha)=\frac{\Phi_{s}^{+} \Phi_{-s}^{-}}{\Phi_{\uparrow}^{-} \Phi_{\downarrow}^{-}+\Phi_{\uparrow}^{-} \Phi_{\downarrow}^{+}+\Phi_{\uparrow}^{+} \Phi_{\downarrow}^{-}}, \\
\left\{l_{s}^{(\infty)}(\alpha)\right\}^{*}=\frac{\Phi_{s}^{-, \chi}}{\Phi_{s}^{-}+\lambda_{0}^{\chi}},
\end{gathered}
$$

and

$$
l_{s}^{b \sigma(\infty)}(\alpha)=\frac{\Gamma_{b \sigma, s}\left(1-f_{b}\left(\omega_{s}\right)\right)-I_{b \sigma}^{\text {Steady }}(\alpha)}{\Phi_{s}^{-}},
$$

with $\omega_{s}=\omega_{0}+s g_{S} B_{S}$. In the following, we fix $\alpha^{\prime \prime}$ to zero bias $\left(\beta_{b}=\beta, \mu_{b}=\mu\right)$ and suppose Eq. (64). Then, $l_{s}^{b \sigma(\infty)}(\alpha)$ equals $v_{s}^{b \sigma}\left(\alpha^{\prime}\right)$ given by Eq. (61) and $\rho_{s}^{(\infty)}(\alpha)$ are given by

$$
\rho\left(s B_{S}\right)=\frac{e^{-\beta\left(\omega_{s}-\mu\right)}}{1+e^{-\beta\left(\omega_{\downarrow}-\mu\right)}+e^{-\beta\left(\omega_{\uparrow}-\mu\right)}} .
$$

We emphasize that $F_{m n}^{b \sigma(\infty)}(\alpha)$ can be obtained by just a replacement,

$$
E_{s}(\alpha)=f\left(\omega_{s}\right) \rightarrow \rho\left(s B_{S}\right),
$$



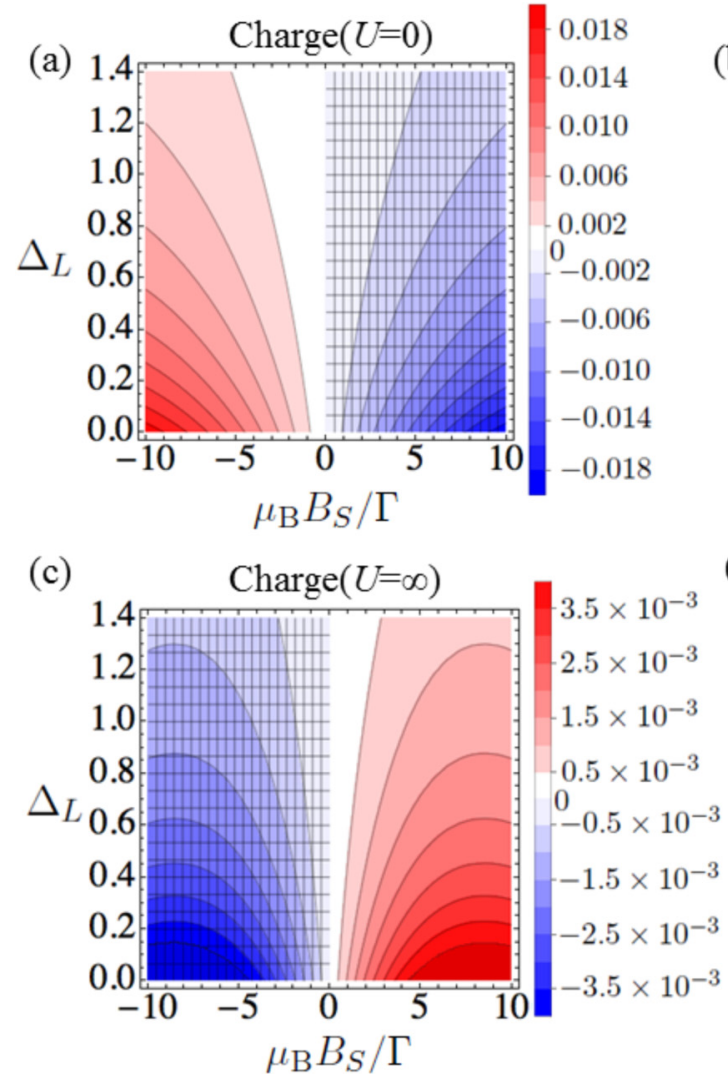

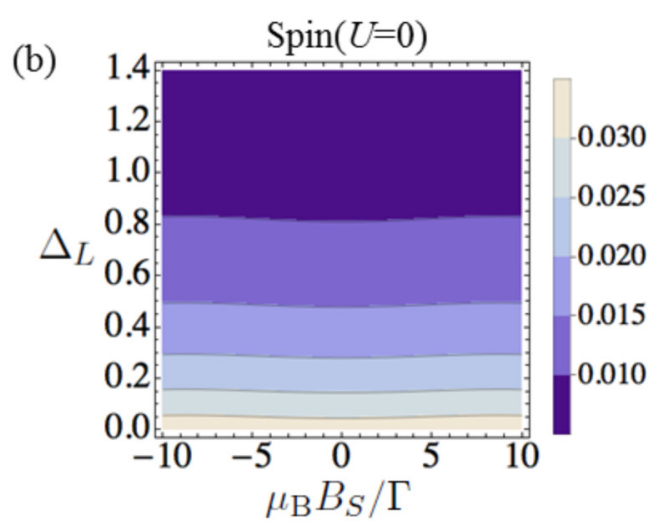

(d)

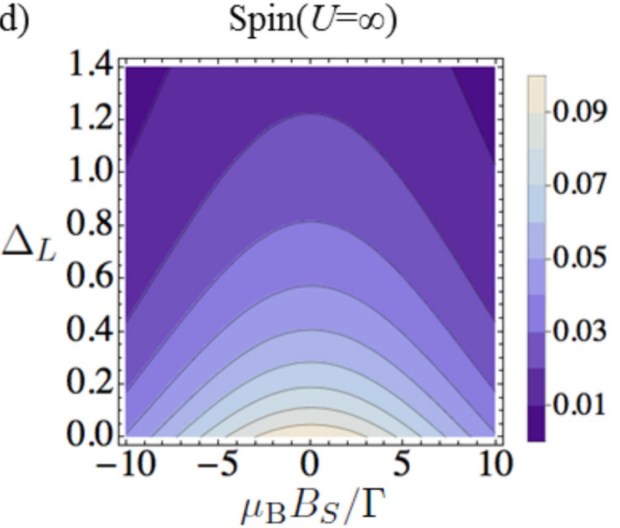

FIG. 2. (Color online) (a) BSN curvature of charge of $\left(\Delta_{L}, B_{S}\right)$ pump, $\left[F_{\Delta_{L}, B_{S}}^{L \uparrow}+F_{\Delta_{L}, B_{S}}^{L \downarrow}\right] / \frac{\mu_{\mathrm{B}}}{\Gamma}$ at $U=0$, (b) the BSN curvature of spin, $\left[F_{\Delta_{L}, B_{S}}^{L \uparrow}-F_{\Delta_{L}, B_{S}}^{L \downarrow}\right] / \frac{\mu_{\mathrm{B}}}{\Gamma}$ at $U=0$, (c) $\left[F_{\Delta_{L}, B_{S}}^{L \uparrow}+F_{\Delta_{L}, B_{S}}^{L \downarrow}\right] / \frac{\mu_{\mathrm{B}}}{\Gamma}$ at $U=\infty$, and (d) $\left[F_{\Delta_{L}, B_{S}}^{L \uparrow}-F_{\Delta_{L}, B_{S}}^{L \downarrow}\right] / \frac{\mu_{\mathrm{B}}}{\Gamma}$ at $U=\infty$. The values of the parameters used for these plots are $\gamma_{L}=\Gamma_{R}=\Gamma, \gamma_{L}^{\prime}=\Gamma_{R}^{\prime}=0.1$, and $B_{L}=0$ and other conditions are the same as Fig. 1. The hatched areas of (a), (c) denote negative value.

in Eq. (63). The charge and spin BSN curvatures of $\left(B_{L}, B_{S}\right),\left(\Delta_{L}, B_{S}\right)$ pump are given by a replacement $f^{\prime}\left(\omega_{0} \pm\right.$ $\left.g_{S} B_{S}\right) \rightarrow \rho^{\prime}\left( \pm B_{S}\right)$ in Eqs. (65) and (66), where $\rho^{\prime}\left(B_{S}\right) \stackrel{\text { def }}{=}$ $\frac{1}{g_{S}} \frac{\partial \rho\left(B_{S}\right)}{\partial B_{S}}$. Similar to $U=0$, the charge and spin BSN curvatures of $\left(B_{L}, B_{R}\right),\left(\Delta_{L}, \Delta_{R}\right)$ pump are zero.

In Figs. 1(a)-1(d), we plot the BSN curvatures of $\left(B_{L}, B_{S}\right)$ pump normalized by $\left(\mu_{\mathrm{B}} / \Gamma\right)^{2}$, where $\Gamma=\Gamma_{L}=\Gamma_{R}$ and $\mu_{\mathrm{B}}=$ $57.88 \mu \mathrm{eV} / \mathrm{T}$ is the Bohr magneton. For $U=0$, the charge and spin BSN curvatures are shown in Fig. 1(a) and Fig. 1(b), and for $U=\infty$ these are shown in Figs. 1(c) and 1(d). The vertical and horizontal axes of these plots are the strength of magnetic fields $B_{S}, B_{L}$ normalized by $\Gamma / \mu_{\mathrm{B}}$. The values of the parameters used for these plots are $\Gamma_{L}=\Gamma_{R}=\Gamma, \Gamma_{L}^{\prime}=\Gamma_{R}^{\prime}=$ $0.1, \beta=0.5 / \Gamma, \omega_{0}=\mu-3 \Gamma, B_{R}=0$, and $g_{L}^{*}=g_{R}^{*}=g_{S}^{*}=$ -0.44 (bulk GaAs). The BSN curvatures of $\left(\Delta_{L}, B_{S}\right)$ pump normalized by $\mu_{\mathrm{B}} / \Gamma$ are shown similarly in Figs. 2(a)-2(d). In all plots, $\gamma_{L}=\Gamma_{R}=\Gamma, \gamma_{L}^{\prime}=\Gamma_{R}^{\prime}=0.1, B_{L}=0$, and other conditions are the same as in Fig. 1. In Figs. 1 and 2, the maximum values of $\left|\Gamma_{b}^{\prime}\left(g_{S} B_{S}-g_{b} B_{b}\right)\right| / \Gamma_{b}$ are 0.44 and 0.22 $(<1)$, respectively. The pumped charges and spins are given by Eq. (67).

Figure 3(a) shows the instantaneous average numbers of the up spin electron of the QD, $n\left(B_{S}\right)=f\left(\omega_{0}+g_{S} B_{S}\right)$ (for $U=0$, solid line) or $\rho\left(B_{S}\right)$ (for $U=\infty$, dashed line) for $\beta=$ $0.5 / \Gamma, \omega_{0}=\mu-3 \Gamma$, and $g_{S}=-0.44 \times \mu_{\mathrm{B}} / 2$. Because two electrons cannot occupy a $\mathrm{QD}$ at $U=\infty$, the magnetic field dependence of $\rho\left(B_{S}\right)$ is more sensitive than $f\left(\omega_{0}+g_{S} B_{S}\right)$. Figures 3(b) and 3(c) show $n^{\prime}\left(B_{S}\right) \mp n^{\prime}\left(-B_{S}\right)$ normalized by $1 / \Gamma$, where $n^{\prime}\left( \pm B_{S}\right)=\left.\frac{1}{g_{S}} \frac{\partial n(B)}{\partial B}\right|_{B= \pm B_{S}}$.

In Figs. 2(a) and 2(c), the charge BSN curvatures of ( $\left.\Delta_{L}, B_{S}\right)$ pump vanish at $B_{S}=0$. This is because the first term of Eq. (66) vanishes since $n^{\prime}\left(B_{S}\right)-n^{\prime}\left(-B_{S}\right)=0$ for $B_{S}=0$ and the second term vanishes since $g_{S} B_{S}-g_{b} B_{b}=0$ for $B_{S}=0=B_{L}$. Similarly, in Figs. 1(b) and 1(d), the spin BSN curvatures of $\left(B_{L}, B_{S}\right)$-pump vanish at $B_{S}=0=B_{L}$. The zero lines in these plots relate to the cancellation between the first and second terms of Eq. (65). Figures 1(a), 1(c) and Figs. 1(b), 1(d) are respectively symmetric and antisymmetric under the transformation $\left(B_{S}, B_{L}\right) \rightarrow\left(-B_{S},-B_{L}\right)$. Similarly, Figs. 2(b), 2(d) and Figs. 2(a), 2(c) are respectively symmetric and antisymmetric under the transformation $B_{S} \rightarrow$ $-B_{S}$. We emphasize that pure charge and pure spin pumps are respectively realized for $\left(B_{L}, B_{S}\right)$ pump and $\left(\Delta_{L}, B_{S}\right)$ pump such that the areas $S^{n}$ in Eq. (67) are symmetric under the above transformations. An instance of symmetric area of $\left(B_{L}, B_{S}\right)$ pump is a disk of which the center is $B_{S}=0=B_{L}$.

In $\omega_{0}>\mu$ region, the larger $\omega_{0}-\mu$, the less difference between $U=0$ and $U=\infty$ becomes. The Coulomb interaction prevents two electrons from occupying the QD. This effect is conspicuous in the $\omega_{0}<\mu$ region, although it is not important in the $\omega_{0}>\mu$ region. 

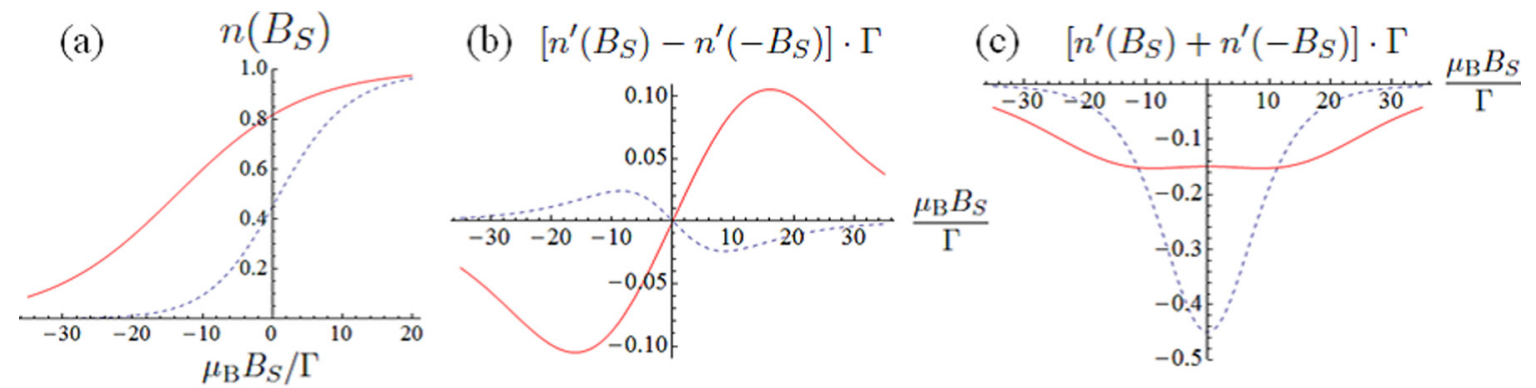

FIG. 3. (Color online) (a) $n\left(B_{S}\right)=f\left(\omega_{0}+g_{S} B_{S}\right)$ (solid line) or $\rho\left(B_{S}\right)$ (dashed line). (b) $n^{\prime}\left(B_{S}\right)-n^{\prime}\left(-B_{S}\right)$ and (c) $n^{\prime}\left(B_{S}\right)+n^{\prime}\left(-B_{S}\right)$, where $n^{\prime}\left( \pm B_{S}\right)=\left.\frac{1}{g_{S}} \frac{\partial n(B)}{\partial B}\right|_{B= \pm B_{S}}$. In all plots, $\beta=0.5 / \Gamma, \omega_{0}=\mu-3 \Gamma$, and $g_{S}=-0.44 \times \mu_{\mathrm{B}} / 2$.

As shown in Figs. 1(a), 1(c) and Figs. 2(b), 2(d), the $B_{S}$ dependence of the charge BSN curvature of $\left(B_{L}, B_{S}\right)$ pump and the spin BSN curvature of $\left(\Delta_{L}, B_{S}\right)$ pump at $U=0$ are more gentle than those at $U=\infty$. It results from the behavior of $n^{\prime}\left(B_{S}\right)+n^{\prime}\left(-B_{S}\right)$ as shown in Fig. 3(c).

As shown in Figs. 1(b), 1(d) and in Figs. 2(a), 2(c), the $B_{S}$ dependence of the spin BSN curvature of $\left(B_{L}, B_{S}\right)$ pump and the charge BSN curvature of $\left(\Delta_{L}, B_{S}\right)$ pump are opposite. This is because the leading term (in weak magnetic field region) of these are proportional to $n^{\prime}\left(B_{S}\right)-n^{\prime}\left(-B_{S}\right)$ and its $B_{S}$ dependence is opposite in $U=0$ and $U=\infty$ for $\omega_{0}-\mu<0$ as indicated in Fig. 3(b). This inversion is realized for only $\omega_{0}-\mu<0$ region. At $\omega_{0}=\mu, f^{\prime}\left(\omega_{0}+\right.$ $\left.g_{S} B_{S}\right)-f^{\prime}\left(\omega_{0}-g_{S} B_{S}\right)$ vanish. In $\omega_{0}>\mu$ region, the signs of $f^{\prime}\left(\omega_{0}+g_{S} B_{S}\right)-f^{\prime}\left(\omega_{0}-g_{S} B_{S}\right)$ and $\rho^{\prime}\left(B_{S}\right)-\rho^{\prime}\left(-B_{S}\right)$ are the same.

In Figs. 1 and 2, absolute values of the normalized BSN curvatures are smaller than unity. However, we can improve this problem by tuning $g$ factors. The first and second terms of the right side of Eq. (65) are the second and third order in the $g$ factors, and the first and second terms of the right side of Eq. (66) are the first and second order in the $g$ factors. If all $g$ factors change to -20 (for example, for the materials like InAs, InSb), the first, second, and third order terms become about 45, 2000, and 90000 times. In fact, for these values of $g$ factors, the assumption Eq. (64) is not appropriate for magnetic fields that are not small; we need concrete energy dependence of linewidth functions.

\section{CONCLUSION}

In this paper, we investigated quantum adiabatic pump of charge and spin using the FCS-QME (full counting statistics with quantum master equation) approach proposed by Yuge et al. [43]. We studied the nonadiabatic effect and showed the correspondence between our approach and the real-time diagrammatic approach [34] (Sec. II B), and confirmed the consistency between the two methods in the concrete model, the one level interacting quantum dot (QD) (Sec. IVC and Sec. VC). Moreover, in Sec. II B, we showed that the Berry-Sinitsyn-Nemenman (BSN) phase derived under the "adiabatic" condition (which makes the Berry phase like treatment appropriate) has the nonadiabatic information. The FCS-QME picks out one higher order nonadiabatic piece of information from the solution of the QME. Particularly, the instantaneous steady state (the zeroth order of the pumping frequency) gives first order response (pumped current). This fact may be related to Ref. [58] which is connected the BSN vector and the liner response theory of the QME.

We generalized the FCS-QME approach to the multicounting field (Sec. II A) and studied the QDs system weakly coupled to leads $(L$ and $R$ ) in Sec. IV and Sec. V. We showed that the pumped charge and spin coming from the instantaneous steady current are not negligible when the thermodynamic parameters (the chemical potentials and the temperatures of leads) are not fixed to zero bias in noninteracting QDs (Sec. IV B) and an interacting QD (Sec. V B). To observe the spin effects, we consider collinear magnetic fields, which relate to spins through the Zeeman effect, with different amplitudes applying to the QDs $\left(B_{S}\right)$ and the leads $\left(B_{L}\right.$ and $B_{R}$ ). We focused on the dynamic parameters $\left(B_{S}, B_{L / R}\right.$, and the coupling strength between QDs and leads, $\left.\Delta_{L / R}\right)$ as control parameters.

In one level QD with the Coulomb interaction $U$, we analytically calculated the BSN curvatures of spin and charge of $\left(B_{L}, B_{S}\right)$ pump and $\left(\Delta_{L}, B_{S}\right)$ pump for the noninteracting limit $(U=0)$ and the strong interaction limit $(U=\infty)$ using the rotating wave approximation (RWA) defined as the long coarse-graining time limit of the coarse-graining approximation (CGA). The difference between $U=0$ and $U=\infty$ appeared through the instantaneous averages of the numbers of the electron with up and down spin in QD. For $\left(B_{L}, B_{S}\right)$ pump, the energy dependences of linewidth functions, which are usually neglected, are essential. Additionally, the adiabatic modulations of $\left(B_{L}, B_{R}\right)$ or $\left(\Delta_{L}, \Delta_{R}\right)$ can pump neither charge nor spin.

In this paper, only $U=0$ and $U=\infty$ limits are studied. In fact, we can analyze finite $U$ based on Eq. (74). This is our future work. Recently, Yoshii and Hayakawa [57] studied adiabatic pump of charge by only the thermodynamic parameters using the same approach in a similar system without magnetic fields (for finite $U$ ). The work (for the thermodynamic parameters) involving the above problem is contrastive to our work (for the dynamic parameters).

\section{ACKNOWLEDGMENTS}

We acknowledge helpful discussions with S. Ajisaka, K. Watanabe, R. Yoshii, H. Hayakawa, and N. Taniguchi. Particularly, we acknowledge Yu Watanabe for discussions about the derivation of Eq. (31). Part of this work is supported by JSPS KAKENHI (Grant No. 26247051). 


\section{APPENDIX A: LIOUVILLE SPACE}

By following correspondence, an arbitrary linear operator (which operates to the Hilbert space) $\bullet=\sum_{n, m}\langle n| \bullet$ $|m\rangle|n\rangle\langle m|$ is mapped to a vector of the Liouville space [42,54], $\left.|\bullet\rangle\rangle=\sum_{n, m}\langle n|\bullet| m\rangle|n m\rangle\right\rangle$ :

$$
\begin{aligned}
|n\rangle\langle m| & \longleftrightarrow|n m\rangle\rangle, \\
\operatorname{Tr}\left(|m\rangle\left\langle n \mid n^{\prime}\right\rangle\left\langle m^{\prime}\right|\right) & \longleftrightarrow\left\langle\left\langle n m \mid n^{\prime} m^{\prime}\right\rangle\right\rangle, \\
\operatorname{Tr}\left(A^{\dagger} B\right) & \longleftrightarrow\langle\langle A \mid B\rangle\rangle, \\
\operatorname{Tr}(\bullet) & \longleftrightarrow\langle\langle 1 \mid \bullet\rangle\rangle .
\end{aligned}
$$

Here, $\{|n\rangle\}$ is an arbitrarily complete orthonormal basis. The inner product of the Liouville space is defined by the Hilbert-Schmidt product [Eq. (A3)]. The Hermitian conjugate of $|\bullet\rangle\rangle$ is defined as $\left\langle\langle\bullet \mid=(|\bullet\rangle\rangle)^{\dagger}=\sum_{n, m}\langle n|\bullet| m\rangle^{*}\langle\langle n m|\right.$. An arbitrary linear superoperator $\hat{J}$ which operates to any operator $(\bullet)$ is mapped to a corresponding operator of the Liouville space $(\breve{J})$ as

$$
|\hat{J} \bullet\rangle\rangle=\check{J}|\bullet\rangle\rangle .
$$

The matrix representation of $\breve{J}$ (or $\hat{J}$ ) is defined by

$$
J_{n m, k l}=\langle\langle n m|\breve{J}| k l\rangle\rangle .
$$

In the main text of this paper, both $\breve{J}$ and $\hat{J}$ are denoted by $\hat{J}$.

Generally, the Liouvillian $\hat{K}^{\chi}$ operates to an operator $\bullet$ as

$$
\begin{gathered}
\hat{K}^{\chi} \bullet=-i\left[H_{S}, \bullet\right]+\hat{\Pi}^{\chi} \bullet, \\
\hat{\Pi}^{\chi} \bullet=\sum_{a} A_{a}^{\chi} \bullet B_{a},
\end{gathered}
$$

where $H_{S}$ is the system Hamiltonian, $\hat{\Pi}^{x}$ is the dissipator like Eq. (B7), and $A_{a}^{\chi}, B_{a}$ are operators. The matrix representation of Eq. (A7) is given by

$$
\begin{aligned}
\sum_{k, l} K_{n m, k l}^{\chi} \bullet k l= & \sum_{k, l}\left[-i\left\{\left(H_{S}\right)_{n k} \delta_{l m}-\delta_{n k}\left(H_{S}\right)_{l m}\right\} \bullet k l\right. \\
& \left.+\left\{\sum_{a}\left(A_{a}^{\chi}\right)_{n k}\left(B_{a}\right)_{l m}\right\} \bullet \bullet_{k l}\right],
\end{aligned}
$$

where $\bullet_{k l}=\langle k|\bullet| l\rangle$. Hence the matrix representation of $\hat{K}^{\chi}$ is given by

$$
\begin{gathered}
K_{n m, k l}^{\chi}=-i H_{n m, k l}+\Pi_{n m, k l}^{\chi}, \\
H_{n m, k l}=\left(H_{S}\right)_{n k} \delta_{l m}-\delta_{n k}\left(H_{S}\right)_{l m},
\end{gathered}
$$

$$
\Pi_{n m, k l}^{\chi}=\sum_{a}\left(A_{a}^{\chi}\right)_{n k}\left(B_{a}\right)_{l m} .
$$

Finally, we consider the current operators defined by Eq. (22). $\hat{K}^{\mu}=\left.\frac{\partial \hat{K}^{\times}(\alpha)}{\partial\left(i_{\chi \mu}\right)}\right|_{\chi=0}$ is given by

$$
\hat{K}^{\mu} \bullet=\sum A_{a}^{\mu} \bullet B_{a} .
$$

Hence the current operators defined by Eq. (22) are given by

$$
W_{\mu}=\sum_{a} B_{a} A_{a}^{\mu} .
$$

\section{APPENDIX B: DETAILS OF FCS-QME}

$\rho_{\mathrm{tot}}^{\chi}(t)(\mathrm{Sec}$. II A) is governed by the modified von Neumann equation [42]:

$$
\frac{d}{d t} \rho_{\mathrm{tot}}^{\chi}(t)=-i\left[H_{\mathrm{tot}}(t), \rho_{\mathrm{tot}}^{\chi}(t)\right]_{\chi},
$$

where $H_{\text {tot }}(t)$ is the total Hamiltonian and $[A, B]_{\chi}=A_{\chi} B-$ $B A_{-\chi}$ with $A_{\chi}=e^{i \sum_{\mu} \chi_{\mu} O_{\mu} / 2} A e^{-i \sum_{\mu} \chi_{\mu} O_{\mu} / 2} \cdot H_{\mathrm{tot}}(t)$ is given by $H_{\text {tot }}(t)=H_{S}(t)+\sum_{b}\left[H_{b}(t)+H_{S b}(t)\right]$, where $H_{S}$ is the system (denoted by $S$ ) Hamiltonian, $H_{b}$ is the Hamiltonian of bath $b$, and $H_{S b}$ is the tunneling interaction Hamiltonian between $S$ and bath $b$. In the following, we suppose Eqs. (43), (44), and (45) for an arbitrary number of leads $(b=1,2, \ldots, M)$. The initial condition of $\rho_{\mathrm{tot}}^{\chi}(t)$ is given by [42] $\rho_{\text {tot }}^{x}(0)=\sum_{\left\{o_{v}\right\}} P_{\left\{o_{v}\right\}} \rho_{\mathrm{tot}}(0) P_{\left\{o_{v}\right\}}$. Here, $\left\{o_{\nu}\right\}$ denotes eigenvalues of $\left\{O_{v}\right\}$ and $P_{\left\{o_{v}\right\}}$ is a projection operator defined by $O_{\mu} P_{\left\{o_{v}\right\}}=o_{\mu} P_{\left\{o_{v}\right\}}, P_{\left\{o_{v}\right\}} P_{\left\{o_{v}^{\prime}\right\}}=P_{\left\{o_{v}\right\}} \prod_{\mu} \delta_{o_{\mu}, o_{\mu}^{\prime}}$, and $P_{\left\{o_{v}\right\}}^{\dagger}=P_{\left\{o_{v}\right\}}$. We suppose $\rho_{\text {tot }}(0)=\rho(0) \otimes \rho_{B}\left(\alpha_{0}\right)$, where

$$
\rho_{B}\left(\alpha_{t}\right)=\bigotimes_{b} e^{-\beta_{b}(t)\left[H_{b}(t)-\mu_{b}(t) N_{b}\right]} / \Xi_{b}\left(\alpha_{t}\right),
$$

with $\Xi_{b}\left(\alpha_{t}\right)=\operatorname{Tr}_{b}\left[e^{-\beta_{b}(t)\left[H_{b}(t)-\mu_{b}(t) N_{b}\right]}\right]$ and $\operatorname{Tr}_{b}$ denotes the trace of lead $b$. Then, $\rho_{\text {tot }}^{\chi}(0)=\rho(0) \otimes \sum_{\left\{o_{v}\right\}} P_{\left\{o_{v}\right\}} \rho_{B}\left(\alpha_{0}\right) P_{\left\{o_{v}\right\}}$ obeys. If all $O_{\mu}$ are given by $\sum_{b, k, \sigma} o_{b k \sigma}^{\mu} c_{b k \sigma}^{\dagger} c_{b k \sigma}$ with real numbers $o_{b k \sigma}^{\mu}, P_{\left\{o_{v}\right\}}$ commutes to $\rho_{B}(0)$ and $\rho_{\text {tot }}^{\chi}(0)=\rho(0) \otimes$ $\rho_{B}\left(\alpha_{0}\right)$ obeys because $\sum_{\left\{o_{v}\right\}} P_{\left\{o_{v}\right\}}=1$.

Now we move to the interaction picture. An operator in the interaction picture corresponding to $A(t)$ is defined by $A^{I}(t)=U_{0}^{\dagger}(t) A(t) U_{0}(t)$ with $\frac{d U_{0}(t)}{d t}=-i\left[H_{S}(t)+\right.$ $\left.\sum_{b} H_{b}(t)\right] U_{0}(t)$ and $U_{0}(0)=1$. The system reduced density operator in the interaction picture is given by $\rho^{I, \chi}(t)=$ $\operatorname{Tr}_{\text {leads }}\left[\rho_{\text {tot }}^{I, \chi}(t)\right]$, where $\rho_{\text {tot }}^{I, \chi}(t)=U_{0}(t) \rho_{\text {tot }}^{\chi}(t) U_{0}^{\dagger}(t)$ and $\operatorname{Tr}_{\text {leads }}$ denotes the trace of lead space. $\rho_{\mathrm{tot}}^{I, \chi}(t)$ is governed by

$$
\frac{d \rho_{\mathrm{tot}}^{I, \chi}(t)}{d t}=-i\left[H_{\mathrm{int}}^{I}(t), \rho_{\mathrm{tot}}^{I, \chi}(t)\right]_{\chi},
$$

with $H_{\text {int }}=\sum_{b} H_{S b}$. Up to the second order perturbation in $H_{\text {int }}$, we obtain

$$
\begin{aligned}
\rho^{I, \chi}\left(t+\tau_{\mathrm{CG}}\right) & =\rho^{I, \chi}(t)-\int_{t}^{t+\tau_{\mathrm{CG}}} d u \int_{t}^{u} d s \operatorname{Tr}_{\text {leads }}\left\{\left[H_{\mathrm{int}}^{I}(u),\left[H_{\mathrm{int}}^{I}(s), \rho^{I, \chi}(t) \rho_{B}\left(\alpha_{t}\right)\right]_{\chi}\right]_{\chi}\right\} \\
& \equiv \rho^{I, \chi}(t)+\tau_{\mathrm{CG}} \hat{L}_{\tau_{\mathrm{CG}}}^{\chi}(t) \rho^{I, \chi}(t),
\end{aligned}
$$


using the large-reservoir approximation $\rho_{\text {tot }}^{I, \chi}(t) \approx \rho^{I, \chi}(t) \otimes \rho_{B}\left(\alpha_{t}\right)$ and $\operatorname{Tr}_{\text {leads }}\left[H_{\text {int }}^{I}(u) \rho_{B}\left(\alpha_{t}\right)\right]=0$. The arbitrary parameter $\tau_{\mathrm{CG}}$ $(>0)$ is called the coarse-graining time. The coarse-graining approximation [50,51] (CGA) is defined by

$$
\frac{d}{d t} \rho^{I, \chi}(t)=\hat{L}_{\tau_{C G}}^{\chi}(t) \rho^{I, \chi}(t) .
$$

If the cycle time of the modulation of control parameters, $\tau$, is much longer than the coarse-graining time $\tau_{\mathrm{CG}}$, the superoperator $\hat{L}_{\tau_{\mathrm{CG}}}^{\chi}(t)$ is described as a function of the set of control parameters at time $t$. In this paper, we suppose $\tau \gg \tau_{\mathrm{CG}}$. Moreover, $\tau_{\mathrm{CG}}$ should be much shorter than the relaxation time of the system, $\tau_{S} \sim \frac{1}{\Gamma}$. On the other hand, $\tau_{S} \ll \tau$ should hold for the adiabatic pump. Hence $\tau_{\mathrm{CG}} \ll \frac{1}{\Gamma} \ll \tau$ should hold.

In the Schrödinger picture, Eq. (B5) is described as

$$
\frac{d \rho^{\chi}(t)}{d t}=-i\left[H_{S}(t), \rho^{\chi}(t)\right]+\hat{\Pi}_{\tau_{\mathrm{CG}}}^{\chi}\left(\alpha_{t}\right) \rho^{\chi}(t) .
$$

Here, the superoperator $\hat{\Pi}_{\tau_{\mathrm{CG}}}^{\chi}(\alpha)$ operates to an operator $\bullet$ as

$$
\begin{aligned}
\hat{\Pi}_{\tau_{\mathrm{CG}}}^{\chi}(\alpha) \bullet & \sum_{b} \sum_{\omega, \omega^{\prime}} \sum_{n, m, s, s^{\prime}}\left[\Phi_{b, n s, m s^{\prime}}^{-}\left(\chi, \tau_{\mathrm{CG}}, \omega, \omega^{\prime}\right) a_{m s^{\prime}}\left(\omega^{\prime}\right) \bullet\left[a_{n s}(\omega)\right]^{\dagger}-\frac{1}{2} \Phi_{b, n s, m s^{\prime}}^{-}\left(\tau_{\mathrm{CG}}, \omega, \omega^{\prime}\right) \bullet\left[a_{n s}(\omega)\right]^{\dagger} a_{m s^{\prime}}\left(\omega^{\prime}\right)\right. \\
& -\frac{1}{2} \Phi_{b, n s, m s^{\prime}}^{-}\left(\tau_{\mathrm{CG}}, \omega, \omega^{\prime}\right)\left[a_{n s}(\omega)\right]^{\dagger} a_{m s^{\prime}}\left(\omega^{\prime}\right) \bullet+\Phi_{b, n s, m s^{\prime}}^{+}\left(\chi, \tau_{\mathrm{CG}}, \omega, \omega^{\prime}\right)\left[a_{m s^{\prime}}\left(\omega^{\prime}\right)\right]^{\dagger} \bullet a_{n s}(\omega) \\
& \left.-\frac{1}{2} \Phi_{b, n s, m s^{\prime}}^{+}\left(\tau_{\mathrm{CG}}, \omega, \omega^{\prime}\right) \bullet a_{n s}(\omega)\left[a_{m s^{\prime}}\left(\omega^{\prime}\right)\right]^{\dagger}-\frac{1}{2} \Phi_{b, n s, m s^{\prime}}^{+}\left(\tau_{\mathrm{CG}}, \omega, \omega^{\prime}\right) a_{n s}(\omega)\left[a_{m s^{\prime}}\left(\omega^{\prime}\right)\right]^{\dagger} \bullet\right] \\
& -i \sum_{b} \sum_{\omega, \omega^{\prime}} \sum_{n, m, s, s^{\prime}}\left[-\frac{1}{2} \Psi_{b, n s, m s^{\prime}}^{-}\left(\tau_{\mathrm{CG}}, \omega, \omega^{\prime}\right)\left[a_{n s}(\omega)\right]^{\dagger} a_{m s^{\prime}}\left(\omega^{\prime}\right)+\frac{1}{2} \Psi_{b, n s, m s^{\prime}}^{+}\left(\tau_{\mathrm{CG}}, \omega, \omega^{\prime}\right) a_{n s}(\omega)\left[a_{m s^{\prime}}\left(\omega^{\prime}\right)\right]^{\dagger}, \bullet\right],
\end{aligned}
$$

where

$$
X^{ \pm}\left(\chi, \tau_{\mathrm{CG}}, \omega, \omega^{\prime}\right)=\frac{e^{\mp i\left(\omega-\omega^{\prime}\right) \tau_{\mathrm{CG}} / 2}}{2 \pi} \int_{-\infty}^{\infty} d \Omega X^{ \pm}(\Omega, \chi) \tau_{\mathrm{CG}} \operatorname{sinc}\left(\frac{\tau_{\mathrm{CG}}(\Omega-\omega)}{2}\right) \operatorname{sinc}\left(\frac{\tau_{\mathrm{CG}}\left(\Omega-\omega^{\prime}\right)}{2}\right),
$$

and $X^{ \pm}\left(\tau_{\mathrm{CG}}, \omega, \omega^{\prime}\right)=X^{ \pm}\left(\chi=0, \tau_{\mathrm{CG}}, \omega, \omega^{\prime}\right)$. Here, $\operatorname{sinc}(x)=\sin x / x \quad$ and $X^{ \pm}(\Omega, \chi)$ denotes one of $\Phi_{b, n s, m s^{\prime}}^{ \pm}(\Omega, \chi)$, $\Psi_{b, n s, m s^{\prime}}^{ \pm}(\Omega, \chi)$, where

$$
\begin{gathered}
\Phi_{b, n s, m s^{\prime}}^{-}(\Omega, \chi)=2 \pi \sum_{k, \sigma} V_{b k \sigma, n s} V_{b k \sigma, m s^{\prime}}^{*}\left[1-f_{b}\left(\varepsilon_{b k \sigma}\right)\right] e^{i \chi_{b \sigma}} e^{i \lambda_{b \sigma} \varepsilon_{b k}} \delta\left(\varepsilon_{b k \sigma}-\Omega\right), \\
\Phi_{b, n s, m s^{\prime}}^{+}(\Omega, \chi)=2 \pi \sum_{k, \sigma} V_{b k \sigma, n s}^{*} V_{b k \sigma, m s^{\prime}} f_{b}\left(\varepsilon_{b k \sigma}\right) e^{-i \chi_{b \sigma}} e^{-i \lambda_{b \sigma} \varepsilon_{b k}} \delta\left(\varepsilon_{b k \sigma}-\Omega\right), \\
\Psi_{b, n s, m s^{\prime}}^{-}(\Omega, \chi)=2 \sum_{k, \sigma} V_{b k \sigma, n s} V_{b k \sigma, m s^{\prime}}^{*}\left[1-f_{b}\left(\varepsilon_{b k \sigma}\right)\right] e^{i \chi_{b \sigma}} e^{i \lambda_{b \sigma} \varepsilon_{b k}} \mathrm{P} \frac{1}{\varepsilon_{b k \sigma}-\Omega}, \\
\Psi_{b, n s, m s^{\prime}}^{+}(\Omega, \chi)=2 \sum_{k, \sigma} V_{b k \sigma, n s}^{*} V_{b k \sigma, m s^{\prime}} f_{b}\left(\varepsilon_{b k \sigma}\right) e^{-i \chi b \sigma} e^{-i \lambda_{b \sigma} \varepsilon_{b k}} \mathrm{P} \frac{1}{\varepsilon_{b k \sigma}-\Omega},
\end{gathered}
$$

with $V_{b k \sigma, n s}=\sqrt{\Delta_{b}} v_{b k \sigma, n s} \cdot \chi_{b \sigma}$ and $\lambda_{b \sigma}$ denote the counting fields for $N_{b \sigma}$ and $\sum_{k} \varepsilon_{b k} c_{b k \sigma}^{\dagger} c_{b k \sigma}$, respectively. $f_{b}(\varepsilon)=$ $\left[\exp \left(\beta_{b}\left(\mu_{b}-\varepsilon\right)\right)+1\right]^{-1}$ is the Fermi distribution function and $\mathrm{P}$ denotes the Cauchy principal value. The eigenvectors $a_{n s}(\omega)$ are given by

$$
a_{n s}(\omega)=\sum_{\alpha, \beta} \delta_{\omega_{\alpha \beta}, \omega}\left|E_{\beta}\right\rangle\left\langle E_{\alpha}\left|a_{n s}\right| E_{\beta}\right\rangle\left\langle E_{\alpha}\right|,
$$

with $\omega_{\alpha \beta}=E_{\alpha}-E_{\beta}$ and $H_{S}\left|E_{\alpha}\right\rangle=E_{\alpha}\left|E_{\alpha}\right\rangle . \omega$ is one of the elements of $\left\{\omega_{\alpha \beta} \mid\left\langle E_{\alpha}\left|a_{n s}\right| E_{\beta}\right\rangle \neq 0{ }^{\exists}(n, s)\right\}$.

We explain the rotating wave approximation (RWA). If $H_{S}$ is time independent, factors $e^{i\left(\omega-\omega^{\prime}\right) t}$ appear in Eq. (B16). The usual RWA [39] is approximating $e^{i\left(\omega-\omega^{\prime}\right) t}$ as $\delta_{\omega, \omega^{\prime}}$ in Eq. (B16). However, if $H_{S}$ is time dependent, the generalization of the RWA is unclear. In this paper, the RWA is defined as the limit $\tau_{\mathrm{CG}} \rightarrow \infty$ of the CGA. In this limit $\left[\tau_{\mathrm{CG}} \cdot \max _{\omega \neq \omega^{\prime}}\left(\left|\omega-\omega^{\prime}\right|\right) \gg 1\right], \quad X^{ \pm}\left(\chi, \tau_{\mathrm{CG}}, \omega, \omega^{\prime}\right) \approx$ $X^{ \pm}(\omega, \chi) \delta_{\omega, \omega^{\prime}}$ holds because of the fact that $\lim _{\tau_{\mathrm{CG}} \rightarrow \infty} \tau_{\mathrm{CG}} \operatorname{sinc} \frac{\tau_{\mathrm{CG}}(\Omega-\omega)}{2} \operatorname{sinc} \frac{\tau_{\mathrm{CG}}\left(\Omega-\omega^{\prime}\right)}{2}=2 \pi \delta_{\omega, \omega^{\prime}} \delta(\Omega-\omega)$.

If $H_{S}$ is time dependent, this RWA is equivalent to usual RWA.

In the Born-Markov approximation (without RWA), Eq. (B16) sometimes violates the non-negativity of $\rho(t)[=$ $\rho^{x=0}(t)$ ] [49]. The QME of the RWA or the CGA is the Lindblad type [see Eq. (B7) at $\chi=0$ ], which guarantees the non-negativity [39]. In QME of the RWA, the diagonal and off-diagonal elements of $\rho(t)$ are decoupled. Sometimes, the decoupling is justified by the superselection rules 
[59-61]: if two states in the system $|m\rangle$ and $|n\rangle$ differ in an observable which is conserved in the total system, unavoidable interactions lead to rapid decay of $\langle m|\rho(t)| n\rangle$. The standard example is the electron number which is conserved for QDs coupled to nonsuperconducting leads. The total spin projection is also conserved for unpolarized or collinearly polarized leads when spins do not flip by the interaction $H_{S b}$. Hence, in our setting, Eqs. (43), (45) with $v_{b k \sigma, n s} \propto \delta_{\sigma, s}$, and Eq. (46), which was used after Sec. IV C, the decoupling between the diagonal and off-diagonal part of $\left\langle m_{\uparrow} m_{\downarrow}|\rho(t)| n_{\uparrow} n_{\downarrow}\right\rangle$ is justified. However, in the general system (discussed until Sec. IV B), the decoupling cannot be justified by the superselection rules. In fact, as explained the following, the CGA can break the decoupling. However, the QME without decoupling is too hard to analyze analytically.

The FCS-QME of the CGA (for finite $\tau_{\mathrm{CG}}$ ) is more difficult to analyze than that of the RWA. For the one level system, Eq. (46), the matrix representation of the Liouvillian of the RWA by the number states $\left\{\left|n_{\uparrow} n_{\downarrow}\right\rangle\right\}$ is block diagonalized to the diagonal part (spanned by $\left\{\left|n_{\uparrow} n_{\downarrow}\right\rangle\left\langle n_{\uparrow} n_{\downarrow}\right|\right\}_{n_{\uparrow}, n_{\downarrow}=0,1}$ ) and the off-diagonal part (spanned by $\left\{\left|n_{\uparrow} n_{\downarrow}\right\rangle\left\langle m_{\uparrow} m_{\downarrow}\right|\right\}_{\left(n_{\uparrow}, n_{\downarrow}\right) \neq\left(m_{\uparrow}, m_{\downarrow}\right)}$ ). The diagonal block is given by Eq. (68) and the off-diagonal block is a $(12 \times 12)$-diagonal matrix. However, one of the CGA has off-diagonal components which permit transitions between diagonal and off-diagonal if spins can flip by tunneling $H_{\text {int }}$. This is consistent with the above discussion about the superselection rules. Even if spins cannot flip, off-diagonal block is not diagonal. Particularly for $U=0$, the Liouvillian does not reduce to a summation of one particle Liouvillian $\left[\hat{K}_{\uparrow}^{\chi}(\alpha) \otimes \hat{1}_{\downarrow}+\hat{1}_{\uparrow} \otimes \hat{K}_{\downarrow}^{\chi}(\alpha)\right]$. The study of differences between the RWA and the CGA is a future work.

Finally, we recognize the Born-Markov approximation (without RWA). From Eq. (B3), we obtain $\rho_{\text {tot }}^{I, \chi}(t)=\rho_{\text {tot }}^{I, \chi}(0)-$ $i \int_{0}^{t} d u\left[H_{\mathrm{int}}^{I}(u), \rho_{\mathrm{tot}}^{I, \chi}(u)\right]_{\chi}$. Substituting this to Eq. (B3), we obtain

$$
\begin{aligned}
\frac{d \rho_{\mathrm{tot}}^{I, \chi}(t)}{d t}= & -i\left[H_{\mathrm{int}}^{I}(t), \rho_{\mathrm{tot}}^{I, \chi}(0)\right]_{\chi} \\
& -\left[H_{\mathrm{int}}^{I}(t), \int_{0}^{t} d u\left[H_{\mathrm{int}}^{I}(u), \rho_{\mathrm{tot}}^{I, \chi}(u)\right]_{\chi}\right]_{\chi} .
\end{aligned}
$$

Moreover, substituting $\rho_{\mathrm{tot}}^{I, \chi}(u)=\rho_{\mathrm{tot}}^{I, \chi}(t)-i \int_{t}^{u} d s\left[H_{\mathrm{int}}^{I}(s)\right.$, $\left.\rho_{\text {tot }}^{I, \chi}(s)\right]_{\chi}$ to Eq. (B14) and using $\rho_{\text {tot }}^{I, \chi}(t) \approx \rho^{I, \chi}(t) \rho_{B}\left(\alpha_{t}\right)$ and $\operatorname{Tr}_{\text {leads }}\left[H_{\text {int }}^{I}(t) \rho_{B}\left(\alpha_{0}\right)\right]=0$, we obtain

$$
\frac{d \rho^{I, \chi}(t)}{d t}=-\int_{0}^{t} d u\left[H_{\mathrm{int}}^{I}(t),\left[H_{\mathrm{int}}^{I}(u), \rho^{I, \chi}(t) \rho_{B}\left(\alpha_{t}\right)\right]_{\chi}\right]_{\chi},
$$

up to the second order perturbation in $H_{\text {int }}$. Because the integrand decay as $e^{-(t-u) / \tau_{B}}$, where $\tau_{B}$ is the relaxation time of the baths, we can replace $\int_{0}^{t} d u$ to $\int_{-\infty}^{t} d u$, and we obtain

$$
\frac{d \rho^{I, \chi}(t)}{d t}=-\int_{0}^{\infty} d s\left[H_{\mathrm{int}}^{I}(t),\left[H_{\mathrm{int}}^{I}(t-s), \rho^{I, \chi}(t) \rho_{B}\left(\alpha_{t}\right)\right]_{\chi}\right]_{\chi} .
$$

This is called the Born-Markov approximation (without RWA). Equations (B15) or (B16) are also derived from
Eq. (B4). In the second term of the right side in the first line of Eq. (B4), we can replace $\int_{t}^{u} d s$ to $\int_{0}^{u} d s$ or $\int_{-\infty}^{u} d s$. After this replacement, by taking $\left.\frac{\partial}{\partial \tau_{\mathrm{CG}}}\right|_{\tau_{\mathrm{CG}}=0}$, we obtain Eqs. (B15) or (B16), respectively. In Eqs. (B15) or (B16) (after moving to the Schrödinger picture), we can replace $\left\{\alpha_{u}\right\}_{0 \leqslant u \leqslant t}$ or $\left\{\alpha_{t-s}\right\}_{0 \leqslant s \leqslant \infty}$ to $\alpha_{t}$ because of $\tau_{B} \ll \tau$.

\section{APPENDIX C: TIME EVOLUTIONS OF $c_{n}^{\chi}(t)$}

In this section, we derive the time evolution equations of $c_{n}^{\chi}(t)$ of Eq. (5). The left hand side of the FCS-QME, $\left.\left.\frac{d}{d t}\left|\rho^{\chi}(t)\right\rangle\right\rangle=\hat{K}^{\chi}\left(\alpha_{t}\right)\left|\rho^{\chi}(t)\right\rangle\right\rangle$, is

$$
\begin{aligned}
\left.\frac{d}{d t}\left|\rho^{\chi}(t)\right\rangle\right\rangle= & \sum_{n}\left\{\frac{d c_{n}^{\chi}(t)}{d t} e^{\Lambda_{n}^{\chi}(t)}\left|\rho_{n}^{\chi}\left(\alpha_{t}\right)\right\rangle\right\rangle \\
& \left.+c_{n}^{\chi}(t) e^{\Lambda_{n}^{\chi}(t)} \lambda_{n}\left(\alpha_{t}\right)\left|\rho_{n}^{\chi}\left(\alpha_{t}\right)\right\rangle\right\rangle \\
& \left.\left.+c_{n}^{\chi}(t) e^{\Lambda_{n}^{\chi}(t)} \frac{d}{d t}\left|\rho_{n}^{\chi}\left(\alpha_{t}\right)\right\rangle\right\}\right\}
\end{aligned}
$$

and the right hand side of the FCS-QME is

$$
\begin{aligned}
\left.\hat{K}^{\chi}\left(\alpha_{t}\right)\left|\rho^{\chi}(t)\right\rangle\right\rangle & \left.=\sum_{n} c_{n}^{\chi}(t) e^{\Lambda_{n}^{\chi}(t)} \hat{K}^{\chi}\left(\alpha_{t}\right)\left|\rho_{n}^{\chi}\left(\alpha_{t}\right)\right\rangle\right\rangle \\
& \left.=\sum_{n} c_{n}^{\chi}(t) e^{\Lambda_{n}^{\chi}(t)} \lambda_{n}\left(\alpha_{t}\right)\left|\rho_{n}^{\chi}\left(\alpha_{t}\right)\right\rangle\right\rangle .
\end{aligned}
$$

Hence we obtain

$$
\left.\sum_{n}\left\{\frac{d c_{n}^{\chi}(t)}{d t} e^{\Lambda_{n}^{\chi}(t)}\left|\rho_{n}^{\chi}\left(\alpha_{t}\right)\right|\right\rangle+c_{n}^{\chi}(t) e^{\Lambda_{n}^{\chi}(t)} \frac{\left.d\left|\rho_{n}^{\chi}\left(\alpha_{t}\right)\right|\right\rangle}{d t}\right\}=0 .
$$

Applying $\left\langle\left\langle l_{m}^{\chi}\left(\alpha_{t}\right)\right|\right.$ to Eq. (C3), and using $\left\langle\left\langle l_{n}^{\chi}(\alpha) \mid \rho_{m}^{\chi}(\alpha)\right\rangle\right\rangle=$ $\delta_{n m}$, we obtain

$$
\frac{d}{d t} c_{m}^{\chi}(t)=-\sum_{n} c_{n}^{\chi}(t) e^{\Lambda_{n}^{\chi}(t)-\Lambda_{m}^{\chi}(t)}\left\langle\left\langle l_{m}^{\chi}\left(\alpha_{t}\right)\right| \frac{\left.d\left|\rho_{n}^{\chi}\left(\alpha_{t}\right)\right\rangle\right\rangle}{d t} .\right.
$$

By the way, the time derivative of Eq. (3), $\hat{K}^{\chi}\left(\alpha_{t}\right)$ $\left.\left.\left.\rho_{n}^{\chi}\left(\alpha_{t}\right)\right\rangle\right\rangle=\lambda_{n}^{\chi}\left(\alpha_{t}\right)\left|\rho_{n}^{\chi}\left(\alpha_{t}\right)\right\rangle\right\rangle$, is

$$
\begin{aligned}
& \frac{d \hat{K}^{\chi}\left(\alpha_{t}\right)}{d t}\left|\rho_{n}^{\chi}\left(\alpha_{t}\right)\right\rangle \mid+\hat{K}^{\chi}\left(\alpha_{t}\right) \frac{\left.d\left|\rho_{n}^{\chi}\left(\alpha_{t}\right)\right|\right\rangle}{d t} \\
& \left.=\frac{d \lambda_{n}^{\chi}\left(\alpha_{t}\right)}{d t}\left|\rho_{n}^{\chi}\left(\alpha_{t}\right)\right\rangle\right\rangle+\lambda_{n}^{\chi}\left(\alpha_{t}\right) \frac{\left.d\left|\rho_{n}^{\chi}\left(\alpha_{t}\right)\right\rangle\right\rangle}{d t} .
\end{aligned}
$$

Applying $\left\langle\left\langle l_{m}^{\chi}\left(\alpha_{t}\right)\right|\right.$ to this equation, we obtain

$$
\begin{gathered}
\left\langle\left\langle l_{m}^{\chi}\left(\alpha_{t}\right)\left|\frac{d \hat{K}^{\chi}\left(\alpha_{t}\right)}{d t}\right| \rho_{n}^{\chi}\left(\alpha_{t}\right)\right\rangle\right\rangle+\lambda_{m}^{\chi}\left(\alpha_{t}\right) \mid\left\langle l_{m}^{\chi}\left(\alpha_{t}\right)\right| \frac{\left.d\left|\rho_{n}^{\chi}\left(\alpha_{t}\right)\right\rangle\right\rangle}{d t} \\
=\frac{d \lambda_{n}^{\chi}\left(\alpha_{t}\right)}{d t} \delta_{m n}+\lambda_{n}^{\chi}\left(\alpha_{t}\right) \mid\left\langle l_{m}^{\chi}\left(\alpha_{t}\right)\right| \frac{\left.d\left|\rho_{n}^{\chi}\left(\alpha_{t}\right)\right\rangle\right\rangle}{d t},
\end{gathered}
$$

and it leads to

$$
\left\langle\left\langle l_{m}^{\chi}\left(\alpha_{t}\right)\right| \frac{\left.d\left|\rho_{n}^{\chi}\left(\alpha_{t}\right)\right\rangle\right\rangle}{d t}=-\frac{\left\langle\left\langle l_{m}^{\chi}\left(\alpha_{t}\right)\left|\frac{d \hat{K}^{\chi}\left(\alpha_{t}\right)}{d t}\right| \rho_{n}^{\chi}\left(\alpha_{t}\right)\right\rangle\right\rangle}{\lambda_{m}^{\chi}\left(\alpha_{t}\right)-\lambda_{n}^{\chi}\left(\alpha_{t}\right)},\right.
$$


for $\lambda_{m}^{\chi}\left(\alpha_{t}\right) \neq \lambda_{n}^{\chi}\left(\alpha_{t}\right)$. Substituting this to Eq. (C4), we obtain

$$
\begin{aligned}
\frac{d c_{m}^{\chi}(t)}{d t}= & -\left\langle\left\langle l_{m}^{\chi}\left(\alpha_{t}\right)\left|\frac{d}{d t}\right| \rho_{m}^{\chi}\left(\alpha_{t}\right)\right\rangle\right\rangle c_{m}^{\chi}(t) \\
& +\sum_{n(\neq m)} c_{n}^{\chi}(t) e^{\Lambda_{n}^{\chi}(t)-\Lambda_{m}^{\chi}(t)} \frac{\left\langle\left\langle l_{m}^{\chi}\left(\alpha_{t}\right)\left|\frac{d \hat{K}^{\chi}\left(\alpha_{t}\right)}{d t}\right| \rho_{n}^{\chi}\left(\alpha_{t}\right)\right\rangle\right\rangle}{\lambda_{m}^{\chi}\left(\alpha_{t}\right)-\lambda_{n}^{\chi}\left(\alpha_{t}\right)} .
\end{aligned}
$$

The above equation can also be written as

$$
\begin{aligned}
\frac{d \tilde{c}_{m}^{\chi}(t)}{d t}= & \sum_{n(\neq m)} \tilde{c}_{n}^{\chi}(t) e^{\Lambda_{n}^{\chi}(t)-\Lambda_{m}^{\chi}(t)+\eta_{m}^{\chi}(t)-\eta_{n}^{\chi}(t)} \\
& \times \frac{\left\langle\left\langle l_{m}^{\chi}\left(\alpha_{t}\right)\left|\frac{d \hat{K}^{\chi}\left(\alpha_{t}\right)}{d t}\right| \rho_{n}^{\chi}\left(\alpha_{t}\right)\right\rangle\right\rangle}{\lambda_{m}^{\chi}\left(\alpha_{t}\right)-\lambda_{n}^{\chi}\left(\alpha_{t}\right)}
\end{aligned}
$$

where $\tilde{c}_{m}^{\chi}(t)=c_{m}^{\chi}(t) e^{\eta_{m}^{\chi}(t)}$ with

$$
\begin{aligned}
\eta_{m}^{\chi}(t) & =\int_{0}^{t} d s\left|\left\langle l_{m}^{\chi}\left(\alpha_{s}\right)\left|\frac{d}{d s}\right| \rho_{m}^{\chi}\left(\alpha_{s}\right)\right\rangle\right\rangle \\
& =\sum_{k} \int_{C} d \alpha^{k}\left\langle\left\langle l_{m}^{\chi}(\alpha)\left|\frac{\partial}{\partial \alpha^{k}}\right| \rho_{m}^{\chi}(\alpha)\right\rangle\right\rangle .
\end{aligned}
$$

Here, $C$ is the trajectory from $\alpha_{0}$ to $\alpha_{t}, \alpha^{k}$ are the $k$ th component of the control parameters, and $\eta_{m}^{\chi}(t)=O(1)$, since $\left\langle\left\langle l_{m}^{\chi}\left(\alpha_{t}\right)\left|\frac{d}{d t}\right| \rho_{m}^{\chi}\left(\alpha_{t}\right)\right\rangle\right\rangle=O(\omega)$ with $\omega=2 \pi / \tau$. In the right hand side of Eq. (C9), the dominant term is $n=0$ if $m \neq 0$ because $\operatorname{Re} \lambda_{0}^{\chi}(\alpha)>\operatorname{Re} \lambda_{n}^{\chi}(\alpha)$. Using $\frac{d \hat{K}^{\chi}\left(\alpha_{t}\right)}{d t}=O(\Gamma \omega)$, $\lambda_{n}^{\chi}\left(\alpha_{t}\right)=O(\Gamma), \quad e^{\eta_{n}^{\chi}(t)}=O(1)$, and $c_{0}^{\chi}(t) e^{\Lambda_{0}^{\chi}}=O(1)$, we obtain

$$
\frac{d \tilde{c}_{m}^{\chi}(t)}{d t}=O\left(e^{-\Lambda_{m}^{\chi}(t)} \omega\right)
$$

and

$$
\begin{aligned}
c_{m}^{\chi}(t) e^{\Lambda_{m}^{\chi}(t)} & =O\left(\omega \int_{0}^{t} d s e^{\Lambda_{m}^{\chi}(t)-\Lambda_{m}^{\chi}(s)}\right) \\
& =O\left(\frac{\omega}{\Gamma}\right) .
\end{aligned}
$$

For $\chi=0$, Eq. (C12) is also derived from

$$
\rho^{a}(t)=\rho(t)-\rho_{0}\left(\alpha_{t}\right)=\sum_{m \neq 0} c_{m}(t) e^{\Lambda_{m}(t)} \rho_{m}\left(\alpha_{t}\right),
$$

and Eqs. (25) and (27).

\section{APPENDIX D: VALIDITY OF THE ADIABATIC EXPANSION}

In the derivation of the QME with CGA, when going from Eq. (B5) to Eq. (B6), we used the following type of approximation:

$$
\begin{aligned}
& \int_{t}^{t+T} d u \int_{t}^{u} d s G\left([\alpha]_{s}^{u} ; s, u ; t\right) \\
& \quad \approx \int_{t}^{t+T} d u \int_{t}^{u} d s G\left(\left[\alpha_{t}\right] ; s, u ; t\right) .
\end{aligned}
$$

Here, $G\left([\alpha]_{s}^{u}, s, u, t\right) \sim e^{-(u-s) / \tau_{B}}$ and $[\alpha]_{s}^{u}=\left(\alpha_{t^{\prime}}\right)_{t^{\prime}=s}^{u}$ is the control parameters' trajectory and $\left[\alpha_{t}\right]$ is the trajectory which $\alpha_{t^{\prime}}=\alpha_{t}\left(s \leqslant t^{\prime} \leqslant u\right)$. Similarly, in the Born-Markov approximation (BM), when going from Eq. (B15) to Eq. (1), we used

$$
\int_{0}^{t} d u G\left([\alpha]_{u}^{t} ; u, t ; t\right) \approx \int_{-\infty}^{t} d u G\left(\left[\alpha_{t}\right] ; u, t ; t\right) .
$$

Considering the corrections of the above approximations, the QME are given by

$$
\begin{gathered}
\left.\frac{d|\rho(t)\rangle\rangle}{d t}=\mathcal{K}(t)|\rho(t)\rangle\right\rangle, \\
\mathcal{K}(t)=\hat{K}\left(\alpha_{t}\right)+\hat{K}^{\prime}(t), \quad \hat{K}^{\prime}(t)=O\left(\Gamma \omega \tau_{X}\right),
\end{gathered}
$$

with $\omega=2 \pi / \tau$ and $\tau_{X}=\tau_{\mathrm{CG}}$ for CGA; $\tau_{X}=\tau_{B}$ for BM. The corrections are also discussed in Ref. [31]. The discussions between Eq. (24) and Eq. (27) are correct after replacing $K\left(\alpha_{t}\right) \rightarrow \mathcal{K}(t), \mathcal{R}\left(\alpha_{t}\right) \rightarrow \tilde{\mathcal{R}}(t)$, and $\rho_{0}\left(\alpha_{t}\right) \rightarrow \tilde{\rho}_{0}(t)$. Here, $\tilde{\rho}_{0}(t)$ and $\tilde{\mathcal{R}}(t)$ are defined by $\left.\mathcal{K}(t)\left|\tilde{\rho}_{0}(t)\right\rangle\right\rangle=0$ and $\tilde{\mathcal{R}}(t) \mathcal{K}(t)=$ $\left.1-\left|\tilde{\rho}_{0}(t)\right\rangle\right\rangle\langle\langle 1|$, respectively. Equation (25) is corrected to

$$
\begin{aligned}
\left.\left|\tilde{\rho}^{a}(t)\right\rangle\right\rangle & =\tilde{\mathcal{R}}(t) \frac{\left.d\left|\tilde{\rho}_{0}(t)\right\rangle\right\rangle}{d t}+\tilde{\mathcal{R}}(t) \frac{\left.d\left|\tilde{\rho}^{a}(t)\right\rangle\right\rangle}{d t} \\
& \left.\left.=\sum_{n=1}^{\infty}\left[\tilde{\mathcal{R}}(t) \frac{d}{d t}\right]^{n}\left|\tilde{\rho}_{0}(t)\right\rangle\right\rangle \equiv \sum_{n=1}^{\infty}\left|\tilde{\rho}^{a(n)}(t)\right\rangle\right\rangle,
\end{aligned}
$$

with $\tilde{\rho}^{a}(t) \stackrel{\text { def }}{=} \rho(t)-\tilde{\rho}_{0}(t)$. The corrections are given by

$$
\tilde{\rho}_{0}=\rho_{0}\left[1+O\left(\omega \tau_{X}\right)\right], \quad \tilde{\mathcal{R}}=\mathcal{R}\left[1+O\left(\omega \tau_{X}\right)\right],
$$

and

$$
\tilde{\rho}^{a(n)}(t)-\rho^{a(n)}(t)=O\left(\left(\frac{\omega}{\Gamma}\right)^{n} \omega \tau_{X}\right) .
$$

Next, we consider the reasonable range of $n$ of $\rho^{a(n)}(t)$. Because $\rho^{a(n)}(t)=O\left(\frac{\omega}{\Gamma}\right)^{n}$ and $\tilde{\rho}_{0}(t)-\rho_{0}\left(\alpha_{t}\right)=O\left(\omega \tau_{X}\right)$, the reasonable range is $n \leqslant n_{\max }$, where $n_{\max }$ is determined by

$$
\left(\frac{\omega}{\Gamma}\right)^{n_{\max }+1}<\omega \tau_{X} \ll\left(\frac{\omega}{\Gamma}\right)^{n_{\max }} .
$$

Let us consider the reasonable concrete values of the parameters in our model (Sec. III): $\omega=10^{p} \mathrm{MHz}, \Gamma=10 \mu \mathrm{eV}=$ $0.116 \mathrm{~K}, 1 / \Gamma=65.8 \mathrm{ps}, \tau_{\mathrm{CG}}=1 \mathrm{ps}$, and $\tau_{B}=0.1 \mathrm{ps}$. These values lead to

$\omega \tau_{\mathrm{CG}}=10^{-6+p}, \quad \omega \tau_{B}=10^{-7+p}, \quad \frac{\omega}{\Gamma}=10^{-4.18+p}$,

and $n_{\max }=\left[\tilde{n}_{\max }\right]$, with

$$
\tilde{n}_{\max }=\frac{-6+p}{-4.18+p}(\mathrm{CGA}), \quad \frac{-7+p}{-4.18+p}(\mathrm{BM}) .
$$

Here, $[n]$ means the biggest integer below $n$. At $p=0, \tilde{n}_{\max }=$ 1.44 (CGA), $1.67(\mathrm{BM})$ and at $p=3, \tilde{n}_{\max }=2.54$ (CGA), $3.39(\mathrm{BM})$. The larger the nonadiabaticity $\left(\frac{\omega}{\Gamma}\right)$, the larger $n_{\max }$ becomes.

\section{APPENDIX E: PROOF OF EQ. (31)}

First, using Eqs. (24) and (23), we obtain $\left\langle\langle 1| W_{\mu}(\alpha) \mathcal{R}(\alpha) \hat{K}(\alpha)=\left\langle\langle 1| W_{\mu}(\alpha)-\lambda_{0}^{\mu}(\alpha)\left\langle\langle 1|\right.\right.\right.$. Next, $\left\langle\left\langle l_{0}(\alpha)\right|\right.$ $=\left\langle\langle 1|, \lambda_{0}(\alpha)=0\right.$, and Eqs. (4) and (22) lead to $\left\langle\left\langle l_{0}^{\mu}(\alpha)\right|\right.$ 
$\hat{K}(\alpha)=\lambda_{0}^{\mu}(\alpha)\left\langle\langle 1|-\left\langle\langle 1| W_{\mu}(\alpha)\right.\right.$. Hence $\left[\left\langle\langle 1| W_{\mu}(\alpha) \mathcal{R}(\alpha)+\right.\right.$ $\left\langle\left\langle l_{0}^{\mu}(\alpha)\right|\right] \hat{K}(\alpha)=0$ and it leads to Eq. (31). To prove Eq. (31) only Eq. (24) is required and $\left.\hat{K}(\alpha) \mathcal{R}(\alpha)=1-\left|\rho_{0}(\alpha)\right\rangle\right\rangle\langle\langle 1|$ is not necessary. Additionally, the pseudoinverse of the real-time diagrammatic approach Eq. (39) satisfies

$$
\sum_{\kappa} R_{\eta \kappa} K_{\kappa \zeta}^{(0)}=\delta_{\eta \zeta}-p_{\eta}^{(0)} \neq \sum_{\kappa} K_{\eta \kappa}^{(0)} R_{\kappa \zeta},
$$

which corresponds to our

$$
\left.\mathcal{R}(\alpha) \hat{K}(\alpha)=1-\left|\rho_{0}(\alpha)\right\rangle\right\rangle\langle\langle 1| \neq \hat{K}(\alpha) \mathcal{R}(\alpha) .
$$

Equation (31) is shown also as follows. Equation (24) and $\langle\langle 1| \hat{K}(\alpha)=0$ lead to $\hat{K}(\alpha) \mathcal{R}(\alpha) \hat{K}(\alpha)=\hat{K}(\alpha)$, which implies

$$
\hat{K}(\alpha) \mathcal{R}(\alpha)=1-|\sigma(\alpha)\rangle\rangle\langle\langle 1|, \quad\langle\langle 1 \mid \sigma(\alpha)\rangle\rangle=1 . \quad(\mathrm{E} 3)
$$

Applying $\langle\langle 1|$ to Eq. (24), we obtain $\langle\langle 1| \mathcal{R}(\alpha) \hat{K}(\alpha)=0$, which is equivalent to

$$
\langle\langle 1| \mathcal{R}(\alpha)=\mathcal{C}(\alpha)\langle\langle 1| .
$$

By the way, differentiating Eq. (4) for $n=0$ by $i \chi_{\mu}$, we obtain

$$
\left\langle\left\langle l_{0}^{\mu}(\alpha)\right| \hat{K}(\alpha)+\left\langle\langle 1| \hat{K}^{\mu}(\alpha)=\left\langle\langle 1| \lambda_{0}^{\mu}(\alpha) .\right.\right.\right.
$$

Applying $\mathcal{R}(\alpha)$ to this equation and using Eqs. (E3) and (E4), we obtain $[47,58]$

$$
\begin{gathered}
\left\langle\left\langle l_{0}^{\mu}(\alpha)\right|=-\left\langle\langle 1| \hat{K}^{\mu}(\alpha) \mathcal{R}(\alpha)+c_{\mu}(\alpha)\langle\langle 1|,\right.\right. \\
c_{\mu}(\alpha)=\mathcal{C}(\alpha) \lambda_{0}^{\mu}(\alpha)+\left\langle\left\langle l_{0}^{\mu}(\alpha) \mid \sigma(\alpha)\right\rangle\right\rangle .
\end{gathered}
$$

Equation (E6) becomes Eq. (31) because of Eq. (22). Particularly, Yuge [58] used

$$
\mathcal{R}(\alpha)=-\lim _{s \rightarrow \infty} \int_{0}^{s} d t e^{\hat{K}(\alpha) t}\left(1-\left|\rho_{0}(\alpha)\right\rangle\right\rangle\langle\langle 1|),
$$

which satisfies Eqs. (E3) and (E4) with $\sigma(\alpha)=\rho_{0}(\alpha), \mathcal{C}(\alpha)=$ 0 , and Eq. (24) (in Ref. [58], $\mathcal{C}(\alpha)$ was incorrectly set to -1 ).
[1] D. J. Thouless, Phys. Rev. B 27, 6083 (1983).

[2] H. Pothier, P. Lafarge, C. Urbina, D. Esteve, and M. H. Devoret, Europhys. Lett. 17, 249 (1992).

[3] S. K. Watson, R. M. Potok, C. M. Marcus, and V. Umansky, Phys. Rev. Lett. 91, 258301 (2003).

[4] A. Fuhrer, C. Fasth, and L. Samuelson, Appl. Phys. Lett. 91, 052109 (2007).

[5] M. Möttönen, J. J. Vartiainen, and J. P. Pekola, Phys. Rev. Lett. 100, 177201 (2008).

[6] M. R. Buitelaar, V. Kashcheyevs, P. J. Leek, V. I. Talyanskii, C. G. Smith, D. Anderson, G. A. C. Jones, J. Wei, and D. H. Cobden, Phys. Rev. Lett. 101, 126803 (2008).

[7] C. Leicht, B. Kaestner, V. Kashcheyevs, P. Mirovsky, T. Weimann, K. Pierz, and H. W. Schumacher, Physica E 42, 911 (2010).

[8] F. Giazotto, P. Spathis, S. Roddaro, S. Biswas, F. Taddei, M. Governale, and L. Sorba, Nature Phys. 7, 857 (2011).

[9] B. Roche, R.-P. Riwar, B. Voisin, E. Dupont-Ferrier, R. Wacquez, M. Vinet, M. Sanquer, J. Splettstoesser, and X. Jehl, Nat. Commun. 4, 1581 (2013).

[10] F. Hoehne, Y. A. Pashkin, O. V. Astafiev, M. Möttönen, J. P. Pekola, and J. S. Tsai, Phys. Rev. B 85, 140504(R) (2012).

[11] S. J. Chorley, J. Frake, C. G. Smith, G. A. C. Jones, and M. R. Buitelaar, Appl. Phys. Lett. 100, 143104 (2012).

[12] L. Fricke, M. Wulf, B. Kaestner, F. Hohls, P. Mirovsky, B. Mackrodt, R. Dolata, T. Weimann, K. Pierz, U. Siegner, and H. W. Schumacher, Phys. Rev. Lett. 112, 226803 (2014).

[13] B. Kaestner and V. Kashcheyevs, Rep. Prog. Phys. 78, 103901 (2015).

[14] P. W. Brouwer, Phys. Rev. B 58, R10135(R) (1998).

[15] J. E. Avron, A. Elgart, G. M. Graf, and L. Sadun, Phys. Rev. B 62, R10618(R) (2000).

[16] A. Andreev and A. Kamenev, Phys. Rev. Lett. 85, 1294 (2000).

[17] Y. Makhlin and A. D. Mirlin, Phys. Rev. Lett. 87, 276803 (2001).

[18] M. L. Polianski, M. G. Vavilov, and P. W. Brouwer, Phys. Rev. B 65, 245314 (2002).
[19] M. Moskalets and M. Büttiker, Phys. Rev. B 64, 201305(R) (2001).

[20] T. A. Shutenko, I. L. Aleiner, and B. L. Altshuler, Phys. Rev. B 61, 10366 (2000).

[21] Y. Wei, J. Wang, and H. Guo, Phys. Rev. B 62, 9947 (2000).

[22] Y. Levinson, O. Entin-Wohlman, and P. Wölfe, Physica A 302, 335 (2001).

[23] T. Aono, Phys. Rev. B 67, 155303 (2003).

[24] T. Aono, Phys. Rev. Lett. 93, 116601 (2004).

[25] A. Schiller and A. Silva, Phys. Rev. B 77, 045330 (2008).

[26] J. Splettstoesser, M. Governale, J. König, and R. Fazio, Phys. Rev. Lett. 95, 246803 (2005).

[27] E. Sela and Y. Oreg, Phys. Rev. Lett. 96, 166802 (2006).

[28] D. Fioretto and A. Silva, Phys. Rev. Lett. 100, 236803 (2008).

[29] J. Splettstoesser, M. Governale, J. König, and R. Fazio, Phys. Rev. B 74, 085305 (2006).

[30] N. Winkler, M. Governale and J. König, Phys. Rev. B 79, 235309 (2009).

[31] F. Cavaliere, M. Governale, and J. König, Phys. Rev. Lett. 103, 136801 (2009).

[32] R.-P. Riwar and J. Splettstoesser, Phys. Rev. B 82, 205308 (2010).

[33] F. Reckermann, J. Splettstoesser, and M. R. Wegewijs, Phys. Rev. Lett. 104, 226803 (2010).

[34] H. L. Calvo, L. Classen, J. Splettstoesser, and M. R. Wegewijs, Phys. Rev. B 86, 245308 (2012).

[35] S. Juergens, F. Haupt, M. Moskalets, and J. Splettstoesser, Phys. Rev. B 87, 245423 (2013).

[36] N. Winkler, M. Governale, and J. König, Phys. Rev. B 87, 155428 (2013).

[37] C. Timm, Phys. Rev. B 77, 195416 (2008).

[38] S. Koller, M. Grifoni, M. Leijnse, and M. R. Wegewijs, Phys. Rev. B 82, 235307 (2010).

[39] H. P. Breuer and F. Petruccione, The Theory of Open Quantum Systems (Oxford University Press, Oxford, 2002). 
[40] L. S. Levitov and G. B. Lesovik, Pis'ma Zh. Eksp. Teor. Fiz. 58, 225 (1993) [JETP Lett. 58, 230 (1993)].

[41] Y. Utsumi, Phys. Rev. B 75, 035333 (2007).

[42] M. Esposito, U. Harbola, and S. Mukamel, Rev. Mod. Phys. 81, 1665 (2009).

[43] T. Yuge, T. Sagawa, A. Sugita, and H. Hayakawa, Phys. Rev. B 86, 235308 (2012).

[44] M. V. Berry, Proc. R. Soc. A 392, 45 (1984).

[45] N. A. Sinitsyn and I. Nemenman, Europhys. Lett. 77, 58001 (2007).

[46] J. Ren, P. Hänggi, and B. Li, Phys. Rev. Lett. 104, 170601 (2010).

[47] T. Sagawa and H. Hayakawa, Phys. Rev. E 84, 051110 (2011).

[48] T. Yuge, T. Sagawa, A. Sugita, and H. Hayakawa, J. Stat. Phys. 153, 412 (2013).

[49] A. Suarez, R. Silbey, and I. Oppenheim, J. Chem. Phys. 97, 5101 (1992).

[50] G. Schaller and T. Brandes, Phys. Rev. A 78, 022106 (2008).
[51] C. Majenz, T. Albash, H. P. Breuer, and D. A. Lidar, Phys. Rev. A 88, 012103 (2013).

[52] C. Uchiyama, Phys. Rev. E 89, 052108 (2014).

[53] K. Watanabe and H. Hayakawa, Prog. Theor. Exp. Phys. (2014) 113A01.

[54] U. Fano, Phys. Rev. 131, 259 (1963).

[55] M. S. Sarandy and D. A. Lidar, Phys. Rev. A 71, 012331 (2005).

[56] X.-Q. Li, J. Luo, Y.-G. Yang, P. Cui, and Y. J. Yan, Phys. Rev. B 71, 205304 (2005).

[57] R. Yoshii and H. Hayakawa, arXiv:1312.3772.

[58] T. Yuge, J. Phys. A: Math. Theor. 47, 505002 (2014).

[59] G.-C. Wick, A. S. Wightman, and E. P. Wigner, Phys. Rev. D 1, 3267 (1970).

[60] W. H. Zurek, Phys. Rev. D 26, 1862 (1982).

[61] D. Giulini, C. Kiefer, and H. D. Zeh, Phys. Lett. A 199, 291 (1995). 